\title{
Laparoscopic versus open approach in gastrectomy for advanced gastric cancer: a systematic review
}

Zhipeng Zhu' ${ }^{1 \dagger}$, Lulu Li ${ }^{1 \dagger}$, Jiuhua $\mathrm{Xu}^{2}$, Weipeng Ye ${ }^{2}$, Junjie Zeng ${ }^{1}$, Borong Chen ${ }^{1}$ and Zhengjie Huang ${ }^{1,2^{*}}$ (D)

\begin{abstract}
Background: Additional studies comparing laparoscopic gastrectomy (LG) versus open gastrectomy (OG) for advanced gastric cancer (AGC) have been published, and it is necessary to update the systematic review of this subject.

Objective: We conducted the meta-analysis to find some proof for the use of LG in AGC and evaluate whether LG is an alternative treatment for AGC.

Method: Randomized controlled trials (RCT) and high-quality retrospective studies (NRCT) compared LG and OG for AGC, which were published in English between January 2010 and May 2019, were search in PubMed, Embase, and Web of Knowledge by three authors independently and thoroughly. Some primary endpoints were compared between the two groups, including intraoperative time, intraoperative blood loss, harvested lymph nodes, first flatus, first oral intake, first out of bed, post-operative hospital stay, postoperative morbidity and mortality, rate of disease recurrence, and 5-year over survival (5-y OS). Besides, considering for this 10-year dramatical surgical material development between 2010 and 2019, we furtherly make the same analysis based on recent studies published between 2016 and 2019.

Result: Thirty-six studies were enrolled in this systematic review and meta-analysis, including 5714 cases in LAG and 6094 cases in OG. LG showed longer intraoperative time, less intraoperative blood loss, and quicker recovery after operations. The number of harvested lymph nodes, hospital mortality, and tumor recurrence were similar. Postoperative morbidity and 5-y OS favored LG. Furthermore, the systemic analysis of recent studies published between 2016 and 2019 revealed similar result.
\end{abstract}

Conclusion: A positive trend was indicated towards LG. LG can be performed as an alternative to OG for AGC. Keywords: Systematic review, Meta-analysis, Gastrectomy, Laparoscopy, Advanced gastric cancer

\footnotetext{
* Correspondence: huangzhengjie@xmu.edu.cn

†Zhipeng Zhu and Lulu Li contributed equally to this work.

'Department of Gastrointestinal Surgery, Xiamen Cancer Center, The First Affiliated Hospital of Xiamen University, 55 Zhen Hai Road, Si Ming District, Xiamen 361003, Fujian, People's Republic of China

${ }^{2}$ Department of Clinical Medicine, Fujian Medical University, Fuzhou 350004,

Fujian, People's Republic of China
}

(C) The Author(s). 2020 Open Access This article is licensed under a Creative Commons Attribution 4.0 International License, which permits use, sharing, adaptation, distribution and reproduction in any medium or format, as long as you give appropriate credit to the original author(s) and the source, provide a link to the Creative Commons licence, and indicate if changes were made. The images or other third party material in this article are included in the article's Creative Commons licence, unless indicated otherwise in a credit line to the material. If material is not included in the article's Creative Commons licence and your intended use is not permitted by statutory regulation or exceeds the permitted use, you will need to obtain permission directly from the copyright holder. To view a copy of this licence, visit http://creativecommons.org/licenses/by/4.0/. The Creative Commons Public Domain Dedication waiver (http://creativecommons.org/publicdomain/zero/1.0/) applies to the data made available in this article, unless otherwise stated in a credit line to the data. 


\section{Introduction}

Gastric cancer is one of the most common malignant diseases worldwide; the incidence and mortality for GC is still high [1]. Surgical resection with lymph node dissection is the most effective treatment for gastric cancer [2]. In the past, conventional open gastrectomy (OG) has been the mainstay of treatment for gastric cancer. Since laparoscopy-assisted gastrectomy was first described in 1994 [3], endoscopic and laparoscopic procedures for early gastric cancer have been increasingly used because of many advantages over OG, including less blood loss, fewer postoperative complications, faster bowel function recovery, shorter hospital stay, and an equivalent longterm outcomes [4-6]. The application of laparoscopic gastrectomy (LG) for advanced gastric cancer (AGC) was first reported [7]. Although several clinical trials have reported the effectiveness of LG $[8,9]$, considering of lacking of long-term oncological outcomes and the technical difficulties, there is no enough evidence to support LG for treating AGC; thus, the use of LG for AGC has been still controversial. Many previous meta-analyses have compared the short-term postoperative outcomes and longterm outcomes $[10,11]$, whereas they have analyzed the results without enough clinical randomized trials and/or with low-quality studies. Many high-quality RCTs related with LG treating AGC have been published recently, especially between 2018 and 2019. Therefore, we conducted this systematic review and meta-analysis to find some proofs for the use of LG in AGC.

\section{Materials and methods}

\section{Search strategy}

The comprehensive publications were identified by searching medical electronic databases PubMed, EMBASE, and Web of Science, which published from July 2010 to May 2019. The following MeSH terms and free-text terms were used: "laparoscopy-assisted gastrectomy", "laparoscopicassisted gastrectomy", "laparoscopy surgery", "laparoscopies", "laparoscopic surgery", "open gastrectomy", "conventional gastrectomy", "stomach neoplasms", "gastric cancer", "gastric neoplasm", and "stomach cancer"; the Boolean operators "AND" and "OR" were used to combined these terms. The references of the relevant articles and previous meta-analysis studies were identified as additional articles. Title and abstracts of each identified article were screened, and the full text of the screened articles was assessed for eligibility. Three authors researched and reviewed independently and thoroughly through the above-mentioned search strategy; the search strategy was provided as supplementary file.

\section{Criteria of inclusion and exclusion}

All included publications in this meta-analysis should meet following criteria:
(1) Clinical studies containing RCTs and NRCTs (case-control study, and cohort study)

(2) Clinical studies having compared LG versus OG for treatment of advanced gastric cancer

(3) Detailed/available data of clinical studies have been reported, including short- or long-term data

(4) Publication in English

All papers containing any of the following criteria were excluded:

(1) Duplicate publication or the publication that did not provide sufficient data

(2) No OG as a control group

(3) Robot-assisted gastrectomy

(4) Abstract only

(5) Gastric surgery performed on benign lesions, nonprimary gastric cancer, or recurrent gastric cancer

(6) Patients in publication had non-curative factors such as distant metastasis of organs

\section{Data extraction}

Clinical data was extracted independently and evaluated critically by two authors. Relevant data included characteristics of included study (author, year of publication, country of publication, study design, study period, male/ female, age, tumor size, BMI, ASA (1:2:3)); summary of laparoscopic technique of included study (type of dissection, type of gastrectomy, retrieved LN, proximal margin, distal margin); systematic review of OS outcomes (follow-up (months), 5-y OS with relevant $P$ ); systematic review of recurrence pattern and sites; surgical outcomes including operative time, intraoperative blood loss, and harvested lymph nodes; recovery outcomes including time to first flatus, time to first oral intake, hospital stay, and mortality (defined as 30-day operative mortality); long-term outcomes including tumor recurrence and 5year OS; and postoperative complications were classified as morbidity, overall complications, specific complications, and general complications. General complications included pneumonia, wound problems, postoperative ileus, and pancreatitis or pancreatic leakage; specific complications included intra-abdominal bleeding, anastomotic bleed, anastomotic stenosis, anastomotic leakage, duodenal stump leakage, abdominal infection, and lymphatic fistula.

\section{Quality assessment}

To assess the quality of included studies, we used the Newcastle-Ottawa Quality Assessment Scale (NOS) for non-RCT [12]. NOS contains 3 categories including selection, comparability, and outcome, which were scaled by eight elements; high-quality elements are awarded by adding a star, no more than one stars could be added into the elements of selection and outcome, 
and no more than two stars could be added into the elements of comparability; then, studies were compared according to the number of stars, total score was 9 stars, 0-5 stars was considered as low-quality and 6-9 stars was considered as high quality. The risk of bias and quality of RCTs were determined by the Jadad scale (JCS) [13]. The high-quality trials should score $\geq 3$ of a maximum possible score of 5 .

\section{Statistical analysis}

STATA 12.0 for Windows was performed for this study. Dichotomous data was calculated by relative risks (RR) with $95 \%$ confidence intervals, and continuous variables were calculated by weighted mean differences (WMD) with $95 \%(\mathrm{CI})$; 5 -year OS was evaluated by pooled hazard ratios (HR) and their 95\% CI. When the HR and 95\% CI were not provided in the studies, some published formula were performed to calculate $\mathrm{HR}$ with $95 \% \mathrm{Cl}$ [14]. A random effect model was used for studies with high heterogeneity, while a fixed-effect model was used for low heterogeneity. $\chi^{2}$ test was used to assess heterogeneity. Funnel plots and Egger's linear regression test were used to assess the publication bias. $P \leq 0.05$ was considered to indicate statistical significance.

\section{Result}

\section{Results of the search and quality assessment}

The study selection process is summarized in the flowchart (Fig. 1). A total of 1220 publications were researched according to the search strategy, eighteen articles were excluded after duplication, and after titles, abstracts, and language were retrieved to assess further, 1140 publications were excluded. Of these studies, twenty-six studies were excluded because they included early gastric cancer only or almost early gastric cancer, were protocols of ongoing studies, were review, and were no control group. In the end, eighteen case-control studies, ten cohort studies, and eight RCT were enrolled in the studies [15-49]. In terms of non-RCT studies, four studies scored 6 (moderate-quality study) on the NOS, and twenty-four studies scored 8-9 (high-quality study) (Table 1). With regard to RCT, two studies scored 4 (high-quality study) on the JCS, and six studies scored 2 (moderate-quality) (Table 2).

\section{Characteristics of included study}

According to the search strategy and criteria of inclusion and exclusion, a total of thirty-six studies published from 2010 to 2019 were eligible for the meta-analysis. A total of 11,808 cases (5714 cases in LAG and 6094 cases in OG) were involved in the study. Among the thirty-six studies, twenty-five studies originated from China, five originated from Japan, four originated from Korea, one originated from Italy, and one originated from Chile. Detailed information for characteristics of included study is shown in Table 3.

\section{Summary of laparoscopic technique}

All the included studies have reported the laparoscopic technique. Thirty-four studies have demonstrated the details on the level of lymphadenectomy, D2 lymphadenectomy was performed in 29 studies, D1, D1+, D2, and $\mathrm{D} 2$ + lymphadenectomy were used in three studies, D1 $+\alpha / \beta$ and D2 were used in one study, and D0, D1, D1 + $\alpha / \beta, D 2$, and $D 2+$ were performed in one study. All the studies have reported the type of gastrectomy; compared with proximal and subtotal gastrectomy, distal and total gastrectomy were frequently used for advanced gastric cancer. Twelve studies reported the resection margin in

Records identified through database searching(1220)

Additional records identified through other sources(0)

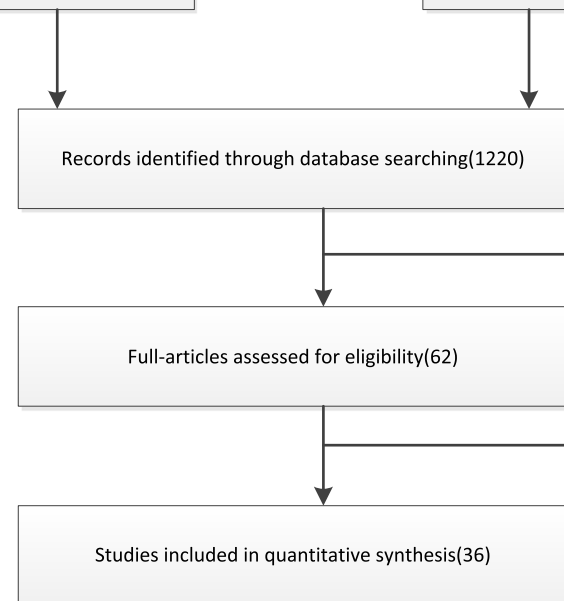

Recoeds excluded(1140)

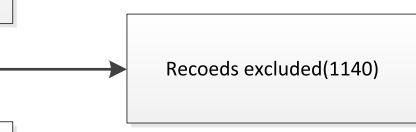

11 only for early gastric cancer(EGC) 4 almost for EGC

5 excluded for no control

3 excluded for review

3 excluded with ongoing RCT

Fig. 1 Flow diagram of the meta-analysis 
Table 1 Assessment of quality of non-RCT studies (NOS)

\begin{tabular}{|c|c|c|c|c|c|c|c|c|c|c|c|}
\hline \multirow[t]{2}{*}{ References } & \multirow[b]{2}{*}{ Year } & \multicolumn{4}{|c|}{ Selection } & \multicolumn{2}{|c|}{ Comparability } & \multicolumn{3}{|c|}{ Outcome } & \multirow[b]{2}{*}{ Score } \\
\hline & & REC & SNEC & $A E$ & $\mathrm{DO}$ & SC & $\mathrm{AF}$ & $\mathrm{AO}$ & $\mathrm{FU}$ & FUO & \\
\hline Zhang et al. [40] & 2017 & * & * & * & * & * & & * & * & * & 8 \\
\hline Xu1 et al. [39] & 2017 & * & $*$ & * & * & $*$ & * & * & * & * & 9 \\
\hline Lu et al. [37] & 2016 & * & * & * & * & * & * & * & * & * & 9 \\
\hline Hao et al. [33] & 2016 & * & * & $*$ & * & * & & * & * & * & 8 \\
\hline Zhang et al. [32] & 2015 & * & * & * & $*$ & * & & * & * & * & 8 \\
\hline Wu et al. [31] & 2015 & * & * & * & * & * & & & & * & 6 \\
\hline Gordon et al. [25] & 2013 & * & * & * & & * & * & * & * & * & 8 \\
\hline Bo et al. [24] & 2013 & * & * & * & * & * & * & * & * & * & 9 \\
\hline Chun et al. [20] & 2012 & * & * & * & * & $*$ & & * & * & * & 8 \\
\hline Chen et al. [19] & 2012 & * & $*$ & * & * & * & * & * & * & * & 9 \\
\hline Zhao et al. [18] & 2011 & * & * & * & * & $*$ & & * & * & * & 8 \\
\hline Shuang et al. [17] & 2011 & * & * & * & * & $*$ & & * & * & * & 8 \\
\hline Shinohara et al. [27] & 2013 & * & * & * & * & * & * & * & * & * & 9 \\
\hline Li3 et al. [42] & 2018 & * & $*$ & * & * & * & * & * & * & * & 9 \\
\hline Chan et al. [45] & 2019 & * & * & * & * & * & * & * & * & * & 9 \\
\hline Fang et al. [28] & 2014 & * & * & * & $*$ & * & * & * & * & * & 9 \\
\hline Xu2 et al. [49] & 2018 & * & * & * & * & * & * & * & * & * & 9 \\
\hline Huang et al. [14] & 2010 & * & $*$ & * & * & $*$ & * & & & & 6 \\
\hline Hamabe et al. [21] & 2012 & * & $*$ & * & * & * & * & * & * & * & 9 \\
\hline Scatizzi et al. [16] & 2011 & * & * & * & * & * & & * & * & * & 8 \\
\hline Inokuchi et al. [41] & 2018 & * & * & * & * & * & * & * & * & * & 9 \\
\hline Li ${ }^{1}$ et al. [35] & 2016 & * & * & * & * & * & * & & & & 6 \\
\hline Zhang et al. [38] & 2016 & * & * & * & * & * & * & * & * & * & 9 \\
\hline Kinoshita et al. [46] & 2019 & $*$ & * & * & $*$ & * & * & * & * & * & 9 \\
\hline Kim et al. [22] & 2012 & * & * & * & & $*$ & * & * & * & * & 8 \\
\hline Moisan et al. [23] & 2012 & * & * & * & * & * & & * & * & * & 8 \\
\hline Qiu et al. [29] & 2014 & * & * & * & * & * & * & & & & 6 \\
\hline Lin et al. [26] & 2013 & $*$ & * & * & * & * & * & * & * & * & 9 \\
\hline
\end{tabular}

REC representativeness of the exposed cohort, SNEC selection of the non-exposed cohort, $A E$ ascertainment of exposure, DO demonstration that outcome of interest was not present at start of study, SC study controls for age and sex, $A F$ study controls for any additional factors, $A O$ assessment of outcome, $F U$ follow-up long enough for outcomes to occur, FUO adequacy of follow-up of cohorts

both LADG and ODG groups, only one article showed there was significant difference in proximal margin between the two groups [21], and all the rest indicated no significant difference between the two groups for proximal margin and distal margins. Thirty-five studies have shown detailed data of retrieved LN between the two groups, thirty-two studies indicated no significant difference, while three studies showed $P<0.05$. Detailed information for characteristics of included studies is shown in Table 4. Relevant pathological characteristics of included studies are shown in Table 5.

\section{Operative results}

Table 6 showed the surgical outcomes of both types of surgery. Twenty-five studies reported the data of intraoperative blood loss [15, 16, 19, 21, 22, 25-28, 30-36, 38, 40, 42, 43, $45-47,49,50]$, twenty studies demonstrated that LG was significantly associated with less blood loss in the operation $[15,19,20,22,25-28,30-35,38,42,45,46,49,50]$, and only one study demonstrated the opposite result [47]. Twenty-nine studies report the data of operative time [15$17,19-22,25-28,30-36,38,40,42-47,49-51]$, the duration of LG was significantly longer than that of OG in twenty-three studies $[15,17,21,22,25,26,28,30-32,34-$ $38,40,42,44,45,47,49-51]$, but only one study demonstrated the opposite result [46]. Twenty-nine studies report the data of retrieved lymph [15-17, 19-22, 25-28, 30-36, $38,40,42-47,49-51]$, twenty-seven have reported the number of retrieved lymph nodes in LG was similar to that in OG $[15-17,19-21,25-28,30,31,33-36,38,40,42-47$, 
Table 2 Assessment of quality of RCTs (Jadad scale)

\begin{tabular}{llllll}
\hline References & Year & Randomization & Blinding & Withdraw and dropout & Jadad's score \\
\hline Wang et al. [48] & 2019 & 2 & 0 & 0 & 2 \\
Lee et al. [47] & 2019 & 2 & 0 & 0 & 2 \\
Shi et al. [44] & 2018 & 2 & 0 & 0 & 2 \\
Park et al. [43] & 2018 & 2 & 0 & 2 & 4 \\
Li1 et al. [35] & 2016 & 2 & 0 & 2 & 4 \\
Hu et al. [34] & 2016 & 2 & 0 & 1 & 1 \\
Cai et al. [15] & 2011 & 1 & 0 & 0 & 2 \\
Cui et al. [30] & 2015 & 2 & 0 & 2 \\
\hline
\end{tabular}

Randomization: randomization was described with appropriate method-2 score, randomization was described without appropriate method- 1 score, no randomization -0 score. Blinding: blinding was performed on all doctors and patients -2 score, blinding was partially performed on doctors and patients -1 score, no blinding -0 score. Withdraw and dropout: the reason of withdraw and dropout was described-1 score, the reason of withdraw and dropout was not described-0 score. Quality: high-quality trials should score $\geq 3$, moderate-quality trials should score $\geq 2$

49-51], and two studies showed that the number of harvested lymph nodes was significantly higher for LG than OG [22, 32]. Our analysis showed that LG could produce satisfactory result, which indicated that lymph node dissection could be carried out with laparoscopic surgery (WMD $=0.02,95 \% \mathrm{CI}=-0.70,0.73 ; P>0.05$; Fig. 2).

Considering for this 10-year dramatical surgical material development between 2010 and 2019, we make a subgroup analysis based on published year (2010-2015 and 2016-2019). Thirteen recent studies published between 2016 and 2019 reported intraoperative blood loss [34-38, $40-42,45-47,49,50]$, ten studies indicated significantly less intraoperative blood loss in LG [34, 35, 38, 41, 42, 45$47,49,50]$, and no study reported opposite result. Recent fourteen studies reported operative time [34-38, 40-42, $44-47,49,50]$, and thirteen studies indicated the duration of LG was significantly longer than that of OG [34-38, 40, 42, 44-47, 49, 50]. All recent fourteen studies revealed that LG was similar to OG in retrieved lymph nodes [34$38,40-42,44-47,49,50]$, and subgroup analysis focused on 2016-2019 demonstrated no significant difference in lymph node dissection $(\mathrm{WMD}=-0.40,95 \% \mathrm{CI}=-1.06$, 0.26; $P>0.05$; Fig. 2). Furthermore, we make a subgroup analysis based on clinical study type; lymph node dissection showed no significant difference between the two groups in the RCT group (WMD $=-0.69,95 \% \mathrm{CI}=-$ 1.45, 0.07; $P>0.05$; Figure S1) and non-RCT (WMD = $0.39,95 \% \mathrm{CI}=-0.55,1.32 ; P<0.05$; Figure $\mathrm{S} 1)$. Besides, we make a subgroup analysis based on the type of gastrectomy; similar lymph node dissection was found between the two groups in distal gastrectomy (DG) $(\mathrm{WMD}=-$ $0.63,95 \% \mathrm{CI}=-1.46,0.21 ; P>0.05$; Figure $\mathrm{S} 2)$ and total gastrectomy $(\mathrm{TG})(\mathrm{WMD}=-1.22,95 \% \mathrm{CI}=-4.70,2.26$; $P>0.05$; Figure $\mathrm{S} 2$ ).

\section{Postoperative recovery}

In terms of postoperative recovery, LG was also superior to OG (Table 7). Twenty-two studies reported a significantly shorter hospital stay after LG than OG [15, $17,19,20,25-28,30,32-36,38,40,42,45,46,49-51]$; four studies showed hospital stay in LG was similar to that in OG [16, 21, 44, 47]. Nineteen demonstrated that first flatus returned earlier after LG with statistical significance $[15,17,19,20,26,27,30,32-36,38,40,43$, $45,47,49,51]$; six studies showed that first flatus in LG was similar to that in OG [16, 21, 25, 42, 44, 50]. Seven studies indicated that first out of bed returned earlier after LG with statistical significance [19, 25, 28, 30, 34, 35, 45]; four studies showed that first out of bed in LG was similar to OG $[16,17,20,49]$. Fourteen individual studies reported a significantly earlier first oral intake after LG than OG $[17,19,20,25,27,28,30,32,34-36$, $42,45,49$ ]; four studies showed that first oral intake in LG was similar to that in OG $[15,16,43,50]$.

In terms of the studies published between 2016 and 2019 , eleven studies indicated significantly shorter hospital stay in LG than OG [34-38, 40-42, 44-47, 49, 50]; three studies reported the similar result between LG and OG [37, 40,44]. Compared with OG, the first flatus returned earlier with statistical significance in recent ten studies for LG [34-38, 40, 41, 45, $47,49]$, and three studies indicated no significant difference between LG and OG [42, 44, 50]. Three significant studies showed that first out of bed returned earlier in LG compared with OG [34, 35, 45], and one study revealed no statistical difference between the two groups [49]. For the first oral intake, six studies demonstrated a significant result for LG [34$36,42,45,49]$, and two studies indicated LG was similar to OG $[37,50]$.

\section{Postoperative morbidity and mortality}

The data from thirty-five studies indicated the rate of overall postoperative complications was lower in LG $(\mathrm{RR}=0.84,95 \% \mathrm{CI}=0.78,0.92, P<0.05)[15-42$, 44-49]; the result was associated with low-grade 


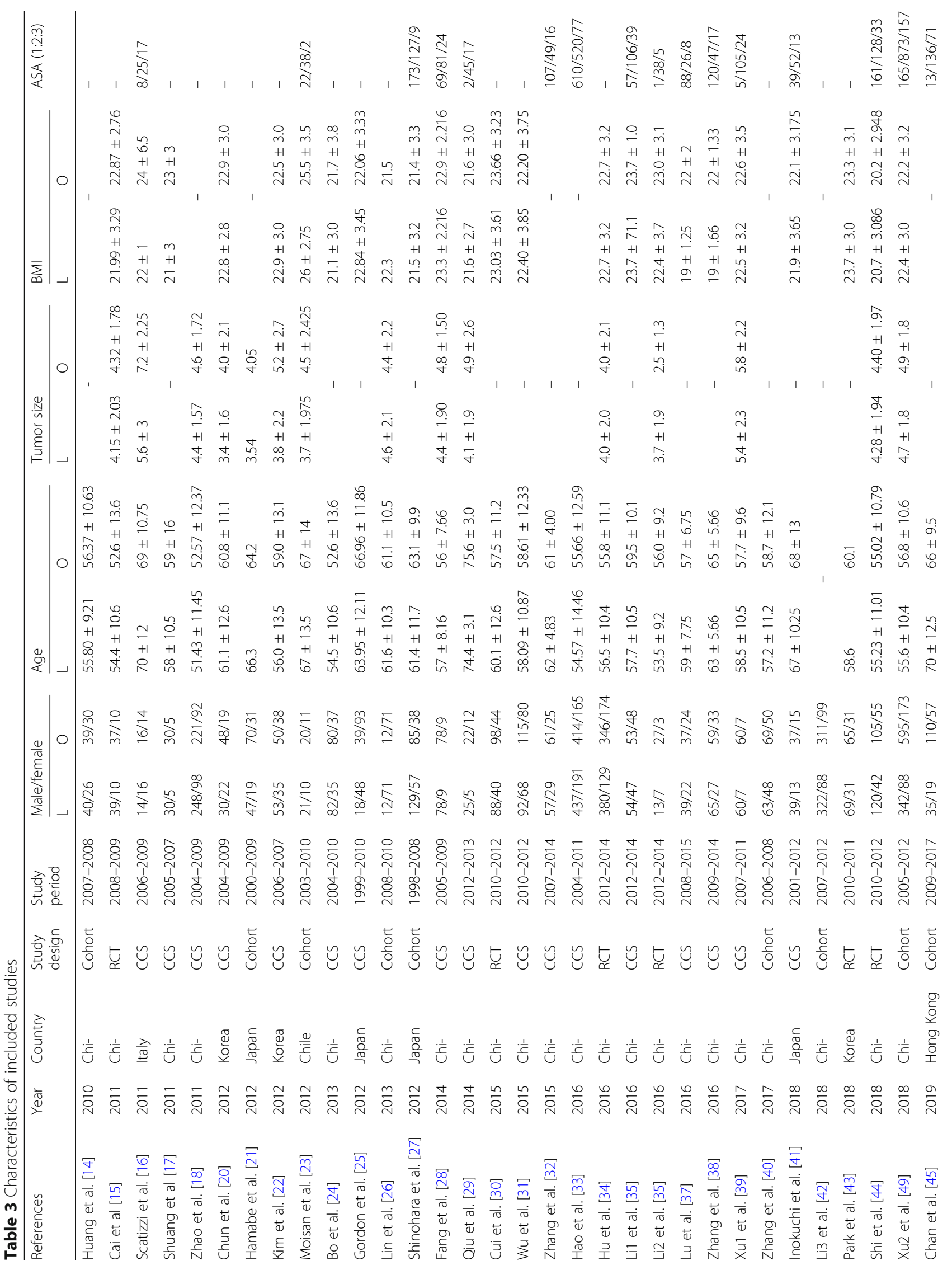




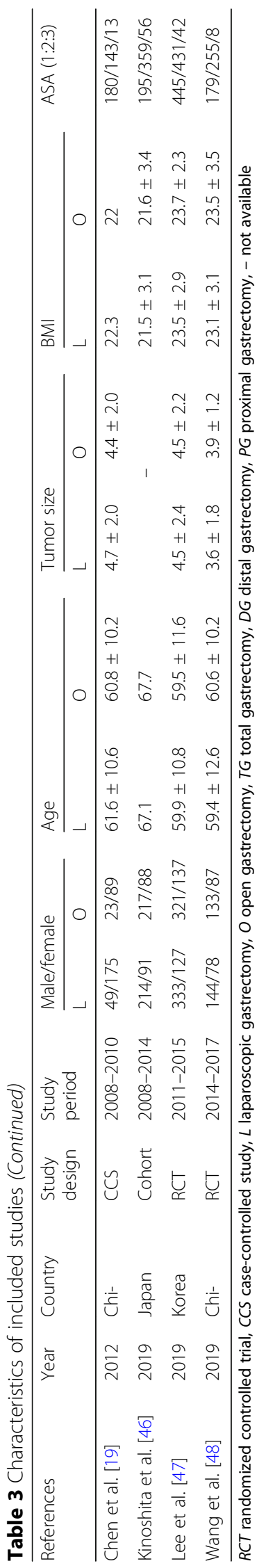




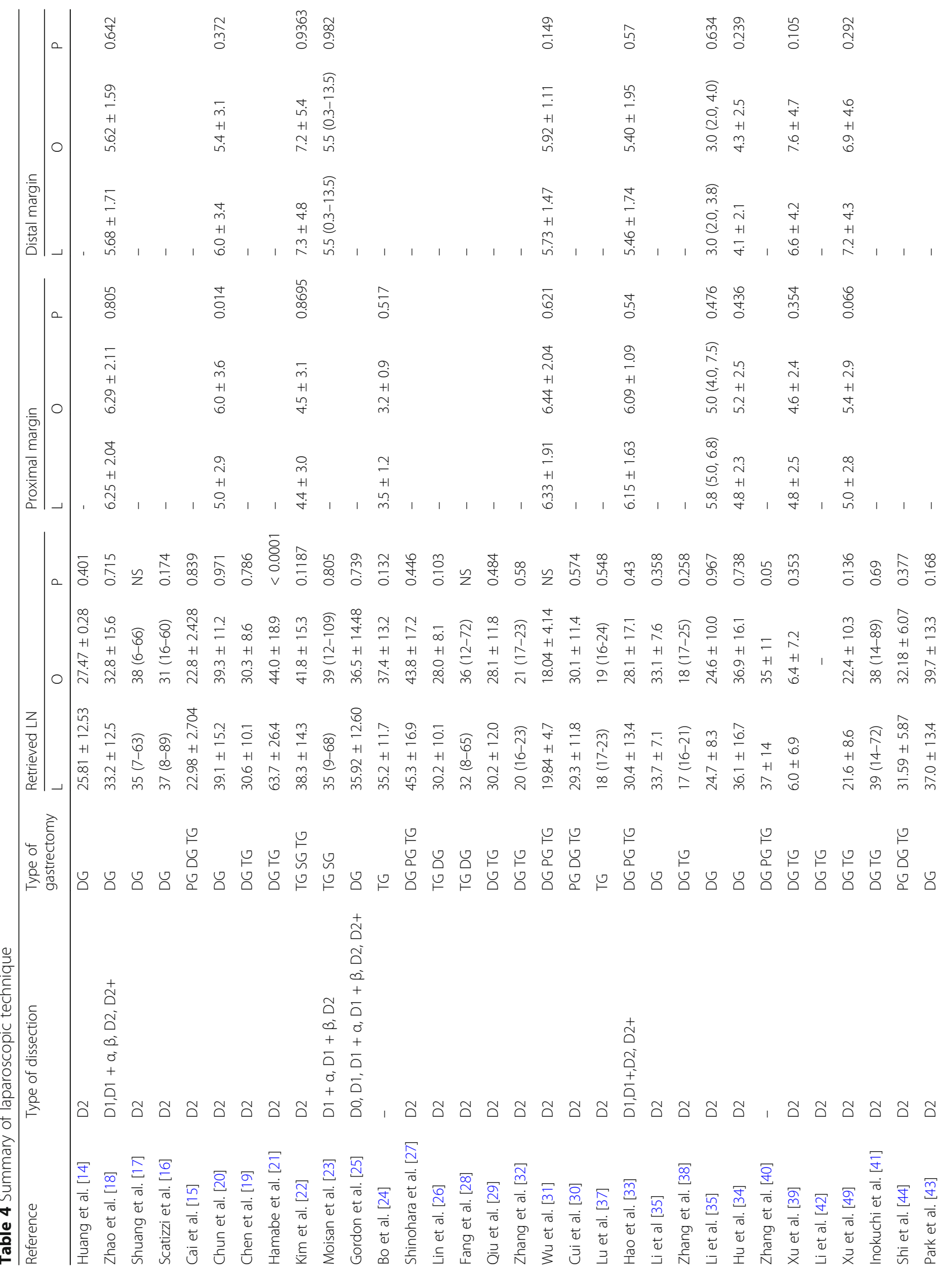




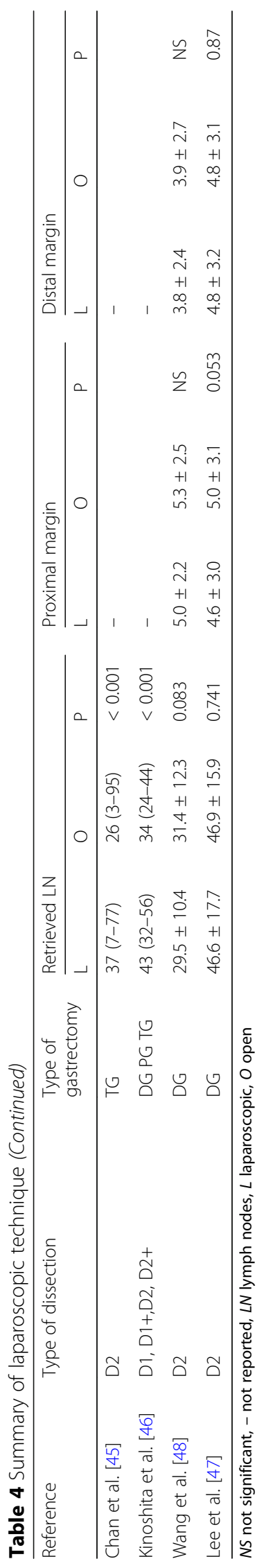




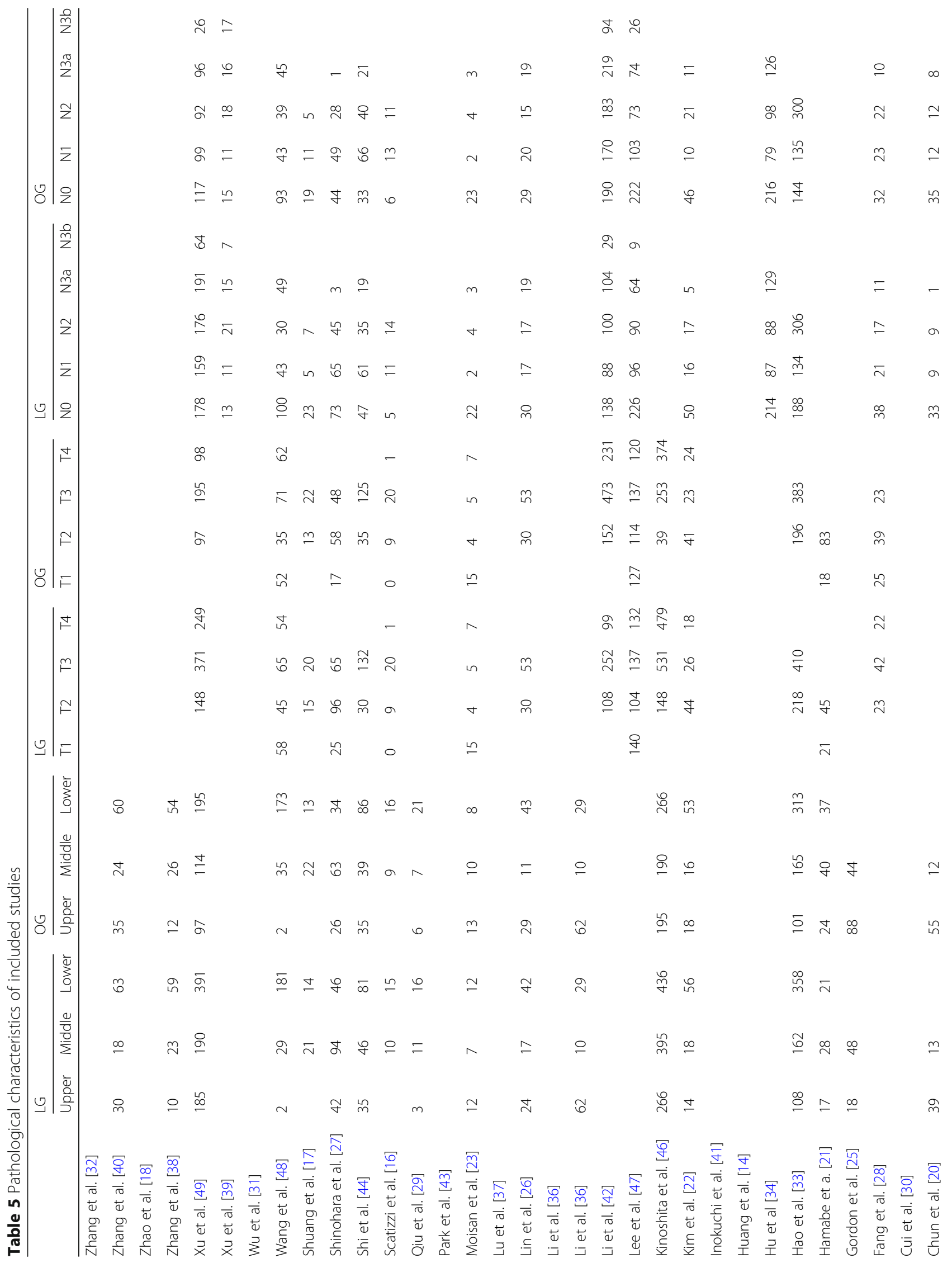




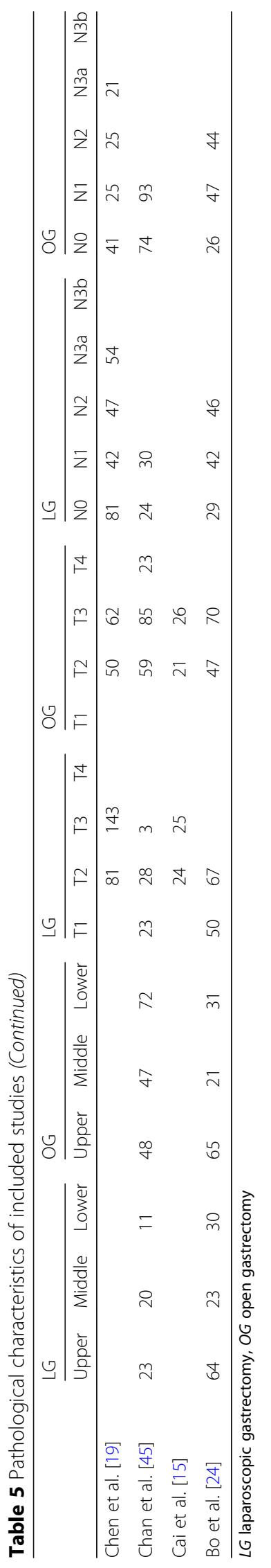


Table 6 Surgical outcomes of LG and OG

\begin{tabular}{|c|c|c|c|c|c|c|}
\hline \multirow[t]{2}{*}{ Reference } & \multicolumn{2}{|c|}{ Blood loss (ml) } & \multicolumn{2}{|c|}{ Operating time (min) } & \multicolumn{2}{|c|}{ Harvested lymph node } \\
\hline & LAG & OG & $\overline{L A G}$ & OG & $\mathrm{LAG}$ & OG \\
\hline \multicolumn{7}{|l|}{ 2010-2015 } \\
\hline Huang et al. [14] & $131.91^{\mathrm{a}}$ & 342.3 & $266.05^{\mathrm{a}}$ & 223.78 & 25.81 & 27.47 \\
\hline Cai et al. [15] & 293.67 & 344.47 & 270.51 & 187.66 & 22.98 & 22.87 \\
\hline Scatizzi et al. [16] & NA & NA & $240^{a}$ & 180 & 31 & 37 \\
\hline Zhao et al. [18] & $128^{\mathrm{a}}$ & 301 & 211 & 204 & 33.2 & 32.8 \\
\hline Chen et al. [19] & $82.7^{\mathrm{a}}$ & 201.7 & 207.2 & 213 & 30.6 & 30.3 \\
\hline Chun et al. [20] & NA & NA & $207.7^{\mathrm{a}}$ & 159.9 & 39.1 & 39.3 \\
\hline Hamabe et al. [21] & $158.3^{\mathrm{a}}$ & 356.3 & $283.1^{\mathrm{a}}$ & 225.9 & $63.7^{\mathrm{a}}$ & 44 \\
\hline Kim et al. [22] & NA & NA & $228.3^{\mathrm{a}}$ & 183.6 & 38.3 & 41.8 \\
\hline Bo et al. [24] & $196.9^{\mathrm{a}}$ & 358.2 & $292.8^{\mathrm{a}}$ & 242.1 & 35.2 & 37.4 \\
\hline Gordon et al. [25] & $107^{\mathrm{a}}$ & 495 & $291^{a}$ & 235 & 35.92 & 36.59 \\
\hline Lin et al. [26] & $78.4^{\mathrm{a}}$ & 200.4 & 212.7 & 226.4 & 30.2 & 28 \\
\hline Shinohara et al. [27] & $154.3^{\mathrm{a}}$ & 388.7 & $369.7^{\mathrm{a}}$ & 263.6 & 45.3 & 43.8 \\
\hline Qiu et al. [29] & $120^{\mathrm{a}}$ & 227.3 & $259.5^{\mathrm{a}}$ & 236.09 & 30.2 & 28.1 \\
\hline Cui et al. [30] & $99^{a}$ & 125 & $258^{\mathrm{a}}$ & 194 & 29.3 & 30.1 \\
\hline Wu et al. [31] & $169.46^{\mathrm{a}}$ & 193. 86 & $228.43^{\mathrm{a}}$ & 207.59 & $19.84^{\mathrm{a}}$ & 18.04 \\
\hline \multicolumn{7}{|l|}{ 2016-2019 } \\
\hline Hao et al. [33] & $154.5^{\mathrm{a}}$ & 311.2 & $257.8^{\mathrm{a}}$ & 231 & 30.4 & 28.1 \\
\hline Hu et al. [34] & $105.5^{\mathrm{a}}$ & 117.3 & $217.3^{a}$ & 186 & 36.1 & 36.9 \\
\hline Li1 et al. [35] & 131.9 & 129.5 & $297.4^{\mathrm{a}}$ & 198.1 & 33.7 & 33.1 \\
\hline Li2 et al. [35] & 94 & 97.9 & $214.2^{\mathrm{a}}$ & 200.3 & 24.7 & 24.6 \\
\hline Lu et al. [37] & $250^{\mathrm{a}}$ & 330 & $240^{\mathrm{a}}$ & 190 & 18 & 19 \\
\hline Xu1 et al. [39] & 322 & 274 & $326^{a}$ & 203 & 24 & 25.6 \\
\hline Zhang et al. [40] & $143^{\mathrm{a}}$ & 223 & 189 & 201 & 37 & 35 \\
\hline Inokuchi et al. [41] & $115^{\mathrm{a}}$ & 420 & $316^{a}$ & 242 & 39 & 38 \\
\hline Park et al. [43] & NA & NA & $257.4^{\mathrm{a}}$ & 183 & 37 & 39.7 \\
\hline Shi et al. [44] & $129^{a}$ & 215.8 & $238.1^{\mathrm{a}}$ & 207.3 & 31.59 & 32.18 \\
\hline Xu2 et al. [49] & $273.7^{\mathrm{a}}$ & 233.6 & $283.8^{\mathrm{a}}$ & 191.5 & 21.6 & 22.4 \\
\hline Chan et al. [45] & $150^{\mathrm{a}}$ & 275 & $321^{a}$ & 365 & 39.5 & 37.5 \\
\hline Lee et al. [47] & $138.3^{\mathrm{a}}$ & 222 & $225.7^{\mathrm{a}}$ & 162.3 & 46.6 & 46.9 \\
\hline Wang et al. [48] & $91.4^{\mathrm{a}}$ & 117.5 & $242.5^{\mathrm{a}}$ & 209.9 & 29.5 & 31.4 \\
\hline
\end{tabular}

$L G$ laparoscopy gastrectomy, OG open gastrectomy

${ }^{\mathrm{a}} P<0.05$

heterogeneity between studies (Fig. 3, Table 8). In terms of the studies published between 2016 and 2019, fifteen studies present data in favor of LG (RR $=0.88,95 \% \mathrm{CI}=0.78,0.99, P<0.05)[34-37,39-42$, $44-47,49,50]$. We furtherly performed a subgroup based on clinical study type, the result favored LG in the non-RCT group ( $\mathrm{RR}=0.82,95 \% \mathrm{CI}=0.74,0.91$, $P<0.05$; Figure S3), and RCT group indicated that LG has similar postoperative complications to OG $(\mathrm{RR}=0.92,95 \% \mathrm{CI}=0.77,1.13, P<0.05$; Figure S3). Moreover, we make a subgroup analysis based on the type of gastrectomy; the result of LG was not inferior to $\mathrm{OG}$ in $\mathrm{TG}(\mathrm{RR}=0.77,95 \% \mathrm{CI}=0.56,1.05, P>$ 0.05 ; Figure S4) and $\mathrm{DG}(\mathrm{RR}=0.82,95 \% \mathrm{CI}=0.68$, 1.00, $P<0.05$; Figure $\mathrm{S} 4$ ).

The subgroup analysis of postoperative complications showed that significantly lower incidence rate of wound problems $(\mathrm{RR}=0.53,95 \% \mathrm{CI}=0.41,0.70 ; P<$ $0.05)$ and postoperative ileus $(\mathrm{RR}=0.64,95 \% \mathrm{CI}=$ $0.43,0.96 ; P<0.05)$ in LG group, and there was no significant difference in other surgery complications, including pneumonia, intra-abdominal bleeding, anastomotic bleed, anastomotic stenosis, anastomotic leakage, duodenal stump leakage, abdominal infection, 


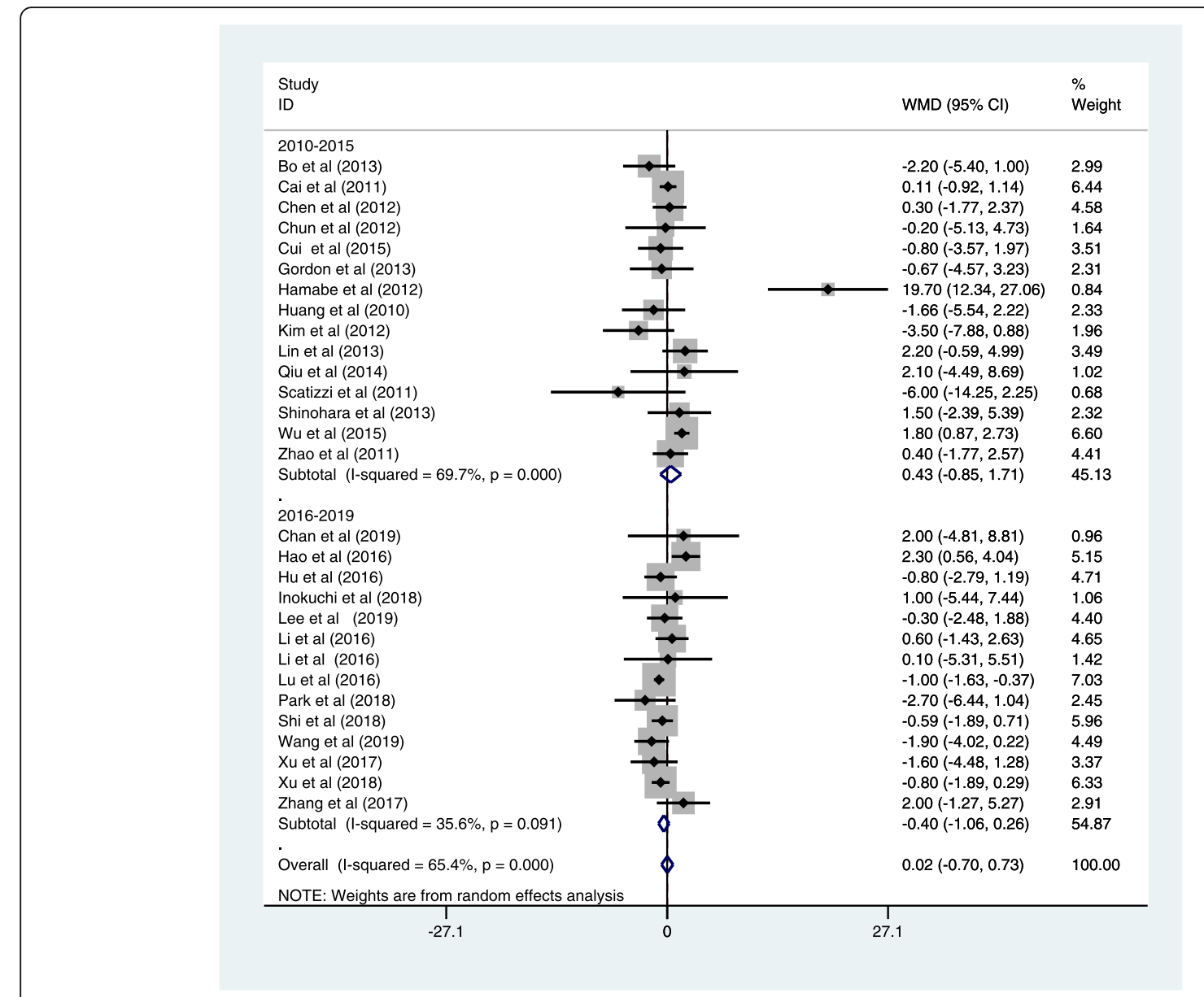

Fig. 2 Forest plot of harvested lymph nodes

lymphatic fistula, pancreatitis, or pancreatic leakage (Table 8). Ten articles reported the post-operative mortality $(\mathrm{RR}=1.27,95 \% \mathrm{CI}=0.57,2.82 ; P>0.05)$ $[19,20,27,28,35,36,40,46-48]$, with no significant difference and heterogeneity among these included articles $\left(I^{2}=0 ; P=0.819\right)$. There were also no significant difference in post-operative mortality between analyzed groups for recent studies $(\mathrm{RR}=1.57,95 \%$ $\mathrm{CI}=0.61,4.05 ; P>0.05$, Fig. 4$)[28,35,36,46,47$, 50]. The subgroup analysis based on clinical study type indicated no significant difference in the nonRCT group $(\mathrm{RR}=1.05,95 \% \mathrm{CI}=0.41,2.67, P>$ 0.05; Fig. 5) and RCT group ( $\mathrm{RR}=1.05,95 \% \mathrm{CI}=$ $0.29,3.80, P>0.05$; Figure S5). The subgroup analysis based on operative procedure also indicated no significant difference in DG ( $R R=0.83,95 \% \mathrm{CI}=0.19$, $3.64, P>0.05$; Figure $\mathrm{S} 6)$ and TG $(\mathrm{RR}=1.19,95 \% \mathrm{CI}$ $=0.08,18.50, P>0.05$; Figure S6).

\section{Long-term postoperative outcomes}

Follow-up ranged widely from 1 month to 149.4 months. Sixteen trials contain the data of 5-year overall survival $[21-23,25,28,29,33,34,38-42$, $46,47]$. The results were in favor of the LG group $(\mathrm{HR}=0.91,95 \% \mathrm{CI}=0.83,0.98 ; P<0.05)$, with moderate grade between the two groups $\left(I^{2}=\right.$ $65.4 \%, P=0.999$, Fig. 5). Thirty-five studies reported no significant difference in the over survival rate. The systematic review of long-term outcomes is summarized in Table 9. We furtherly make a subgroup analysis based on published years. As for the studies published in 2010-2015, there was no significant difference in terms of 5 -year overall survival $(\mathrm{HR}=0.88,95 \% \mathrm{CI}=0.71,1.06 ; P<0.05)[21-23$, $25,28,29,33]$. However, studies published in 2016-2020 revealed LG was associated with better result $(\mathrm{HR}=0.90,95 \% \mathrm{CI}=0.82,0.99 ; P<0.05)$ $[34,38-42,46,47]$. Subgroup analysis based on operative procedure also indicated no significant difference between LG and OG in DG $(\mathrm{RR}=0.86$, $95 \% \mathrm{CI}=0.62,1.10, P>0.05$; Figure S7). There was only one study in the TG group, and it also did not report a significant difference $(R R=0.75,95 \%$ $\mathrm{CI}=0.18,1.77$; Figure S7). 
Table 7 Recovery outcomes of LG and OG

\begin{tabular}{|c|c|c|c|c|c|c|c|c|}
\hline \multirow[t]{2}{*}{ Reference } & \multicolumn{2}{|c|}{ Hospital stay (days) } & \multicolumn{2}{|c|}{ First flatus (days) } & \multicolumn{2}{|c|}{ First out of bed (days) } & \multicolumn{2}{|c|}{ First oral intake } \\
\hline & LAG & OG & $\overline{L A G}$ & OG & LAG & OG & $\mathrm{LAG}$ & OG \\
\hline \multicolumn{9}{|l|}{$2010-2015$} \\
\hline Huang et al. [14] & $9.2^{\mathrm{a}}$ & 11.35 & $3.18^{\mathrm{a}}$ & 4.5 & NA & NA & 6.53 & 7.64 \\
\hline Cai et al. [15] & 11.6327 & 11.4255 & 3.89 & 4.2128 & 4.7755 & 4.8936 & 6.8571 & 6.4681 \\
\hline Scatizzi et al. [16] & $7^{\mathrm{a}}$ & 9 & $2^{\mathrm{a}}$ & 3 & 1 & 1 & $3^{\mathrm{a}}$ & 4 \\
\hline Zhao et al. [18] & $7.9^{\mathrm{a}}$ & 10.7 & $3^{\mathrm{a}}$ & 3.9 & $3^{a}$ & 4.3 & $3.5^{\mathrm{a}}$ & 4.5 \\
\hline Chen et al. [19] & $13.3^{a}$ & 17.4 & $2.6^{a}$ & 3.2 & 2.7 & 2.9 & $4.7^{\mathrm{a}}$ & 5.1 \\
\hline Chun et al. [20] & 7 & 7 & 3.1 & 3.1 & NA & NA & NA & NA \\
\hline Kim et al. [22] & $7^{\mathrm{a}}$ & 10.4 & $3.2^{\mathrm{a}}$ & 3.7 & NA & NA & NA & NA \\
\hline Bo et al. [24] & $7.4^{\mathrm{a}}$ & 10.7 & 3.4 & 3.9 & $3.1^{\mathrm{a}}$ & 5.3 & $4.5^{\mathrm{a}}$ & 5.3 \\
\hline Gordon et al. [25] & $8.4^{\mathrm{a}}$ & 18.1 & $2.7^{\mathrm{a}}$ & 3.8 & NA & NA & NA & NA \\
\hline Lin et al. [26] & $14.2^{\mathrm{a}}$ & 17.2 & $2.9^{\mathrm{a}}$ & 4 & NA & NA & $4.1^{\mathrm{a}}$ & 5.5 \\
\hline Shinohara et al. [27] & $16.3^{a}$ & 24.3 & NA & NA & $2^{a}$ & 3.2 & $3.4^{\mathrm{a}}$ & 5.7 \\
\hline Qiu et al. [29] & $13^{\mathrm{a}}$ & 16.9 & $2.9^{a}$ & 4.6 & $1.2^{\mathrm{a}}$ & 4.1 & $4.5^{\mathrm{a}}$ & 5.5 \\
\hline Wu et al. [31] & $9.44^{a}$ & 11.07 & $3.72^{\mathrm{a}}$ & 4.41 & NA & NA & $5.66^{\mathrm{a}}$ & 7.09 \\
\hline \multicolumn{9}{|l|}{ 2016-1019 } \\
\hline Hao et al. [33] & $7.6^{\mathrm{a}}$ & 10.7 & $3.3^{\mathrm{a}}$ & 3.9 & $3.1^{\mathrm{a}}$ & 4.5 & $3.7^{\mathrm{a}}$ & 4.5 \\
\hline Hu et al. [34] & $10.8^{a}$ & 11.3 & $1.4^{\mathrm{a}}$ & 3.6 & $2.3^{\mathrm{a}}$ & 2.4 & $5.5^{\mathrm{a}}$ & 6 \\
\hline Li1 et al. [35] & $10.5^{a}$ & 11.9 & $2.8^{\mathrm{a}}$ & 3.6 & NA & NA & $3.8^{\mathrm{a}}$ & 4.6 \\
\hline Li2 et al. [35] & 10.875 & 10.625 & $3.2^{\mathrm{a}}$ & 3.9 & NA & NA & 6.357 & 6.25 \\
\hline Lu et al. [37] & $8^{\mathrm{a}}$ & 10 & $2^{\mathrm{a}}$ & 4 & NA & NA & NA & NA \\
\hline Xu1 et al. [39] & 10.7 & 10.2 & $4.4^{\mathrm{a}}$ & 4.8 & NA & NA & NA & NA \\
\hline Zhang et al. [40] & $8.6^{\mathrm{a}}$ & 13.2 & $2.3^{\mathrm{a}}$ & 3.5 & NA & NA & NA & NA \\
\hline Inokuchi et al. [41] & $9^{a}$ & 12 & 3 & 4 & NA & NA & $2^{a}$ & 4 \\
\hline Park et al. [43] & 9.8 & 9.1 & 2.6 & 2.5 & NA & NA & NA & NA \\
\hline Shi et al. [44] & $7.51^{\mathrm{a}}$ & 10.49 & $3.14^{\mathrm{a}}$ & 3.96 & $3.15^{\mathrm{a}}$ & 4.37 & $3.57^{\mathrm{a}}$ & 4.41 \\
\hline Xu2 et al. [49] & $8.2^{\mathrm{a}}$ & 8.7 & $4^{\mathrm{a}}$ & 4.4 & NA & NA & NA & NA \\
\hline Chan et al. [45] & $9^{a}$ & 11 & NA & NA & NA & NA & NA & NA \\
\hline Lee et al. [47] & $8.1^{\mathrm{a}}$ & 9.1 & 3.5 & 3.7 & NA & NA & 3.7 & 3.8 \\
\hline Wang et al. [48] & $9.9^{\mathrm{a}}$ & 10.9 & $2.8^{\mathrm{a}}$ & 3.1 & 1.2 & 1.4 & $7^{\mathrm{a}}$ & 7.9 \\
\hline
\end{tabular}

LG laparoscopy gastrectomy, OG open gastrectomy ${ }^{\mathrm{a}} P<0.05$

No statistical difference was found between the LG and OG groups in tumor recurrence $(\mathrm{RR}=0.93,95 \%$ $\mathrm{CI}=0.81,1.07 ; P>0.05)[19,21-24,28,29,34,39$, $40,46,47]$, with moderate-grade heterogeneity $\left(I^{2}=\right.$ 62.2\%; $P=0.002$ ) (Fig. 6). Besides, we analyzed the data from studies published in 2016-2019; there showed no significant difference between LG and OG $(\mathrm{RR}=0.94,95 \% \mathrm{CI}=0.67,1.31 ; P>0.05)[19,21-$ 24, 28, 29]. Furthermore, subgroup analysis based on operative procedure also indicated no significant difference between LG and OG in DG (RR $=0.94,95 \%$ $\mathrm{CI}=0.79,1.10, P>0.05$; Figure S8). There was only one study in the TG group, and it showed lower incidence rate of tumor recurrence in $L G(R R=0.33$,
$95 \% \mathrm{CI}=0.14,0.78$; Figure S8). In the studies reporting the site of recurrence, local recurrence was the most frequent recurrence site among these common sites; relevant data related with specific recurrent sites are shown in Table 10.

\section{Discussion}

Between 2010 and 2019, there are many high-quality RCTs that have been published. However, many previous meta-analyses have included low-quality studies or a limited number of studies [52, 53]. Thus, we conducted the meta-analysis to evaluate whether LG is an alternative treatment for AGC. Some primary endpoints were compared between LG and OG, including intraoperative 


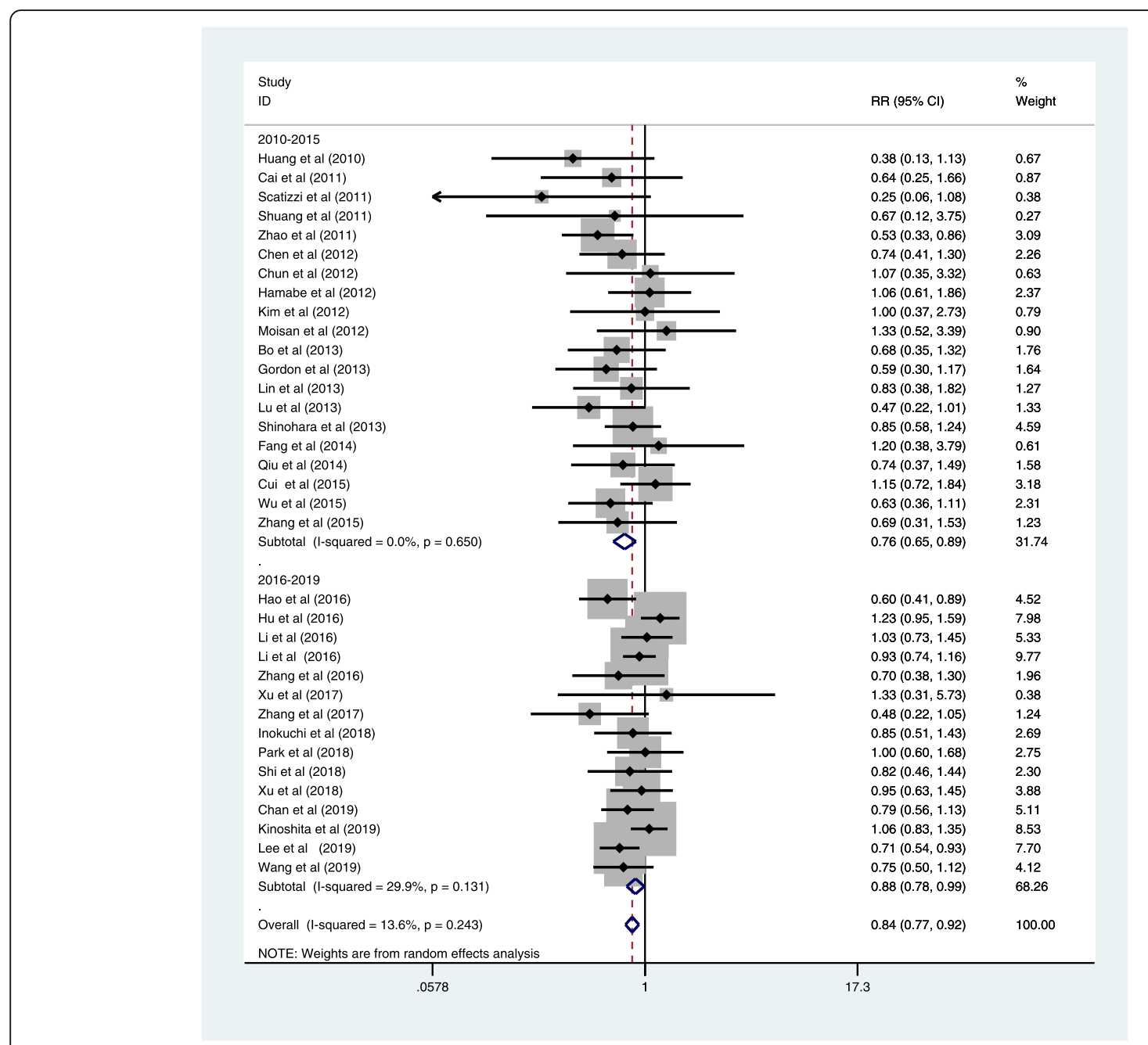

Fig. 3 Forest plot of morbidity

Table 8 Analysis of postoperative morbidity

\begin{tabular}{|c|c|c|c|c|c|c|c|c|c|}
\hline \multirow[b]{2}{*}{ Measure outcomes } & \multicolumn{7}{|l|}{ Sample size } & \multicolumn{2}{|c|}{ Heterogeneity test } \\
\hline & No. of studies & LG & OG & $\mathrm{OR}$, WMD, or HR & Lower $95 \% \mathrm{Cl}$ & Upper $95 \% \mathrm{Cl}$ & $P$ & $\overline{P^{2}(\%)}$ & $P$ \\
\hline Over morbidity & 35 & 79 & 1031 & 0.83 & 0.77 & 0.91 & $>0.05$ & 14.50 & 0.228 \\
\hline \multicolumn{10}{|l|}{ Specific complications } \\
\hline Intra-abdominal bleeding & 12 & 23 & 28 & 0.86 & 0.5 & 1.47 & $>0.05$ & 0 & 0.838 \\
\hline Anastomotic bleed & 12 & 14 & 13 & 1.14 & 0.59 & 2.22 & $>0.05$ & 0 & 0.919 \\
\hline Anastomotic stenosis & 11 & 19 & 24 & 0.75 & 0.42 & 1.31 & $>0.05$ & 0 & 0.747 \\
\hline Anastomotic leakage & 24 & 75 & 97 & 0.85 & 0.63 & 1.15 & $>0.05$ & 0 & 0.819 \\
\hline Duodenal stump leakage & 13 & 27 & 29 & 0.93 & 0.56 & 1.52 & $>0.05$ & 0 & 0.845 \\
\hline Abdominal infection & 16 & 56 & 69 & 0.78 & 0.55 & 1.1 & $>0.05$ & 0 & 0.996 \\
\hline Lymphatic fistula & 8 & 16 & 17 & 0.86 & 0.45 & 1.64 & $>0.05$ & 0 & 0.896 \\
\hline \multicolumn{10}{|l|}{ General complications } \\
\hline Pneumonia & 20 & 106 & 132 & 0.84 & 0.65 & 1.08 & $>0.05$ & 0 & 0.88 \\
\hline Wound problems & 22 & 79 & 143 & 0.53 & 0.41 & 0.7 & $<0.0001$ & 3.50 & 0.413 \\
\hline Postoperative ileus & 15 & 35 & 57 & 0.64 & 0.43 & 0.96 & $<0.05$ & 0 & 0.89 \\
\hline Pancreatitis or pancreatic leakage & 14 & 64 & 42 & 1.42 & 0.98 & 2.05 & $>0.05$ & 0 & 0.935 \\
\hline
\end{tabular}




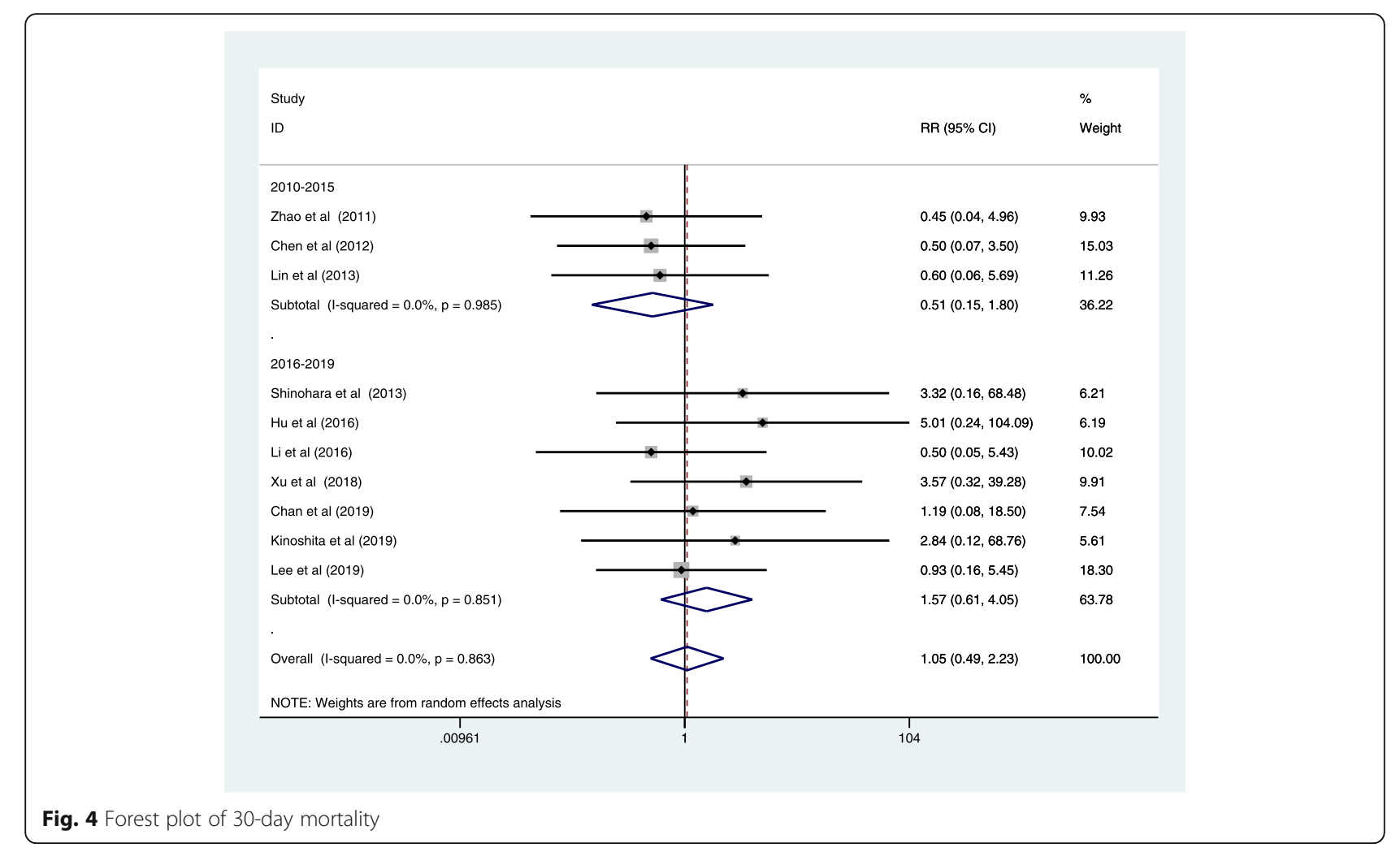

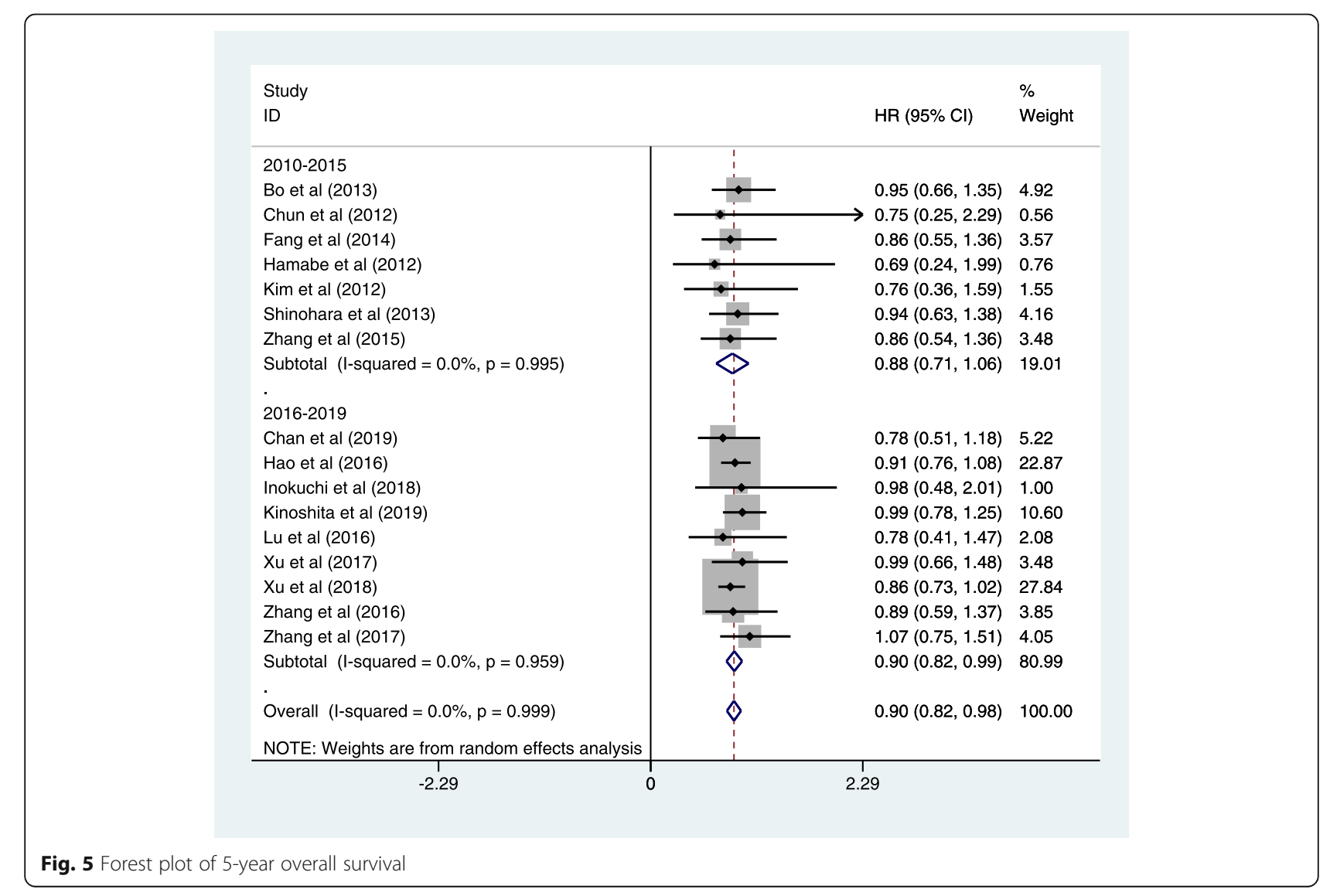


Table 9 Systematic review of OS outcomes

\begin{tabular}{|c|c|c|c|c|}
\hline References & Group & Follow-up (mo.) & OS & $P$ \\
\hline \multirow[t]{2}{*}{ Zhao et al. [18] } & L & $37(6-72)$ & 1 y, 87.2\%; 3 y, 57.2\%; 5 y, 50.3\% & NS \\
\hline & O & & 1 y, 87.1\%; 3 y, $54.1 \% ; 5$ у $49.2 \%$ & \\
\hline \multirow[t]{2}{*}{ Shuang et al. [17] } & L & $36.5(23-50)$ & 50 mo., 64\% & NS \\
\hline & O & $38.5(27-50)$ & 50 mo., $60 \%$ & \\
\hline \multirow[t]{2}{*}{ Scatizzi et al. [16] } & L & $18(2-37)$ & 42 mo., $70.91 \%$ & 0.449 \\
\hline & $\mathrm{O}$ & $18(7-42)$ & 42 mo., $56.77 \%$ & \\
\hline \multirow[t]{2}{*}{ Cai et al. [15] } & L & $22.1354(4-36)$ & 40 mo., $67.1 \%$ & - \\
\hline & $\mathrm{O}$ & & 40 mo., 53.8\% & \\
\hline \multirow[t]{2}{*}{ Chun et al. [20] } & L & $60.4(7.0-91.7)$ & $5 y, 91.3 \%$ & 0.613 \\
\hline & O & $53.2(1.0-82.2)$ & $5 y, 88.6 \%$ & \\
\hline \multirow[t]{2}{*}{ Chen et al. [19] } & L & $19(1-48)$ & $1 \mathrm{y}, 91.5 \%$ & 0.297 \\
\hline & $\mathrm{O}$ & & $1 \mathrm{y}, 89.8 \%$ & \\
\hline \multirow[t]{2}{*}{ Hamabe et al. [21] } & L & $30.4(1-60.9)$ & $5 y, 94.4 \%$ & 0.4877 \\
\hline & $\mathrm{O}$ & $53.5(1.3-111.3)$ & $5 y, 78.5 \%$ & \\
\hline \multirow[t]{2}{*}{ Kim et al. [22] } & L & $53.7(8.3-138.1)$ & $5 y, 85.9 \%$ & 0.463 \\
\hline & $\mathrm{O}$ & $58.1(0.3-106.2)$ & $5 y, 83.1 \%$ & \\
\hline \multirow[t]{2}{*}{ Moisan et al. [23] } & L & $28 \mathrm{mo}$ & $3 y, 82.3 \%$ & 0.557 \\
\hline & $\mathrm{O}$ & 40 mo. & $3 y, 86.9$ & \\
\hline \multirow[t]{2}{*}{ Gordon et al. [25] } & L & $49.2(4-146)$ & $5 y, 79.2 \%$ & NS \\
\hline & $\mathrm{O}$ & & $5 y, 77.2 \%$ & \\
\hline \multirow[t]{2}{*}{ Bo et al. [24] } & L & 61.2 mo. (6-84 mo.) & $5 y, 49.3 \%$ & 0.756 \\
\hline & O & & $5 y, 46.5 \%$ & \\
\hline \multirow[t]{2}{*}{ Shinohara et al. [27] } & L & $48.8(25-58.5)$ & $5 y, 68.1 \%$ & 0.968 \\
\hline & $\mathrm{O}$ & & $5 y, 63.7 \%$ & \\
\hline \multirow[t]{2}{*}{ Lin et al. [26] } & L & $23.0(12-50)$ & $1 \mathrm{y}, 88.0 \%$ & NS \\
\hline & O & & $1 \mathrm{y}, 85.5 \%$ & \\
\hline \multirow[t]{2}{*}{ Fang et al. [28] } & L & $44(1-82)$ & $5 y, 59 \%$ & 5.525 \\
\hline & $\mathrm{O}$ & & $5 y, 54 \%$ & \\
\hline \multirow[t]{2}{*}{ Zhang et al. [32] } & L & 38 & $5 y, 59 \%$ & 0.523 \\
\hline & $\mathrm{O}$ & 40 & $5 y, 56 \%$ & \\
\hline \multirow[t]{2}{*}{ Hao et al. [33] } & L & 53.5 & $5 y, 57.65 \%$ & 0.22 \\
\hline & $\mathrm{O}$ & & 5 y, 53.69\% & \\
\hline \multirow[t]{2}{*}{ Li1 et al. [35] } & L & - & - & - \\
\hline & $\mathrm{O}$ & - & - & \\
\hline \multirow[t]{2}{*}{ Zhang et al. [38] } & L & 38 & $5 y, 57 \%$ & 0.606 \\
\hline & $\mathrm{O}$ & 40 & $5 y, 50 \%$ & \\
\hline \multirow[t]{2}{*}{ Zhang et al. [40] } & L & $37(3-60)$ & 1 y, $89.2 \% ; 3$ y, $72.1 \% ; 5$ y, 45.7\% & NS \\
\hline & $\mathrm{O}$ & & $1 y, 87.4 \% ; 3 y, 68.1 \% ; 5 y, 42.3 \%$ & \\
\hline \multirow[t]{2}{*}{ Xu et al. [39] } & L & $22(3-100)$ & $5 y, 31.3 \%$ & 0.949 \\
\hline & $\mathrm{O}$ & & $5 y, 29.9 \%$ & \\
\hline \multirow[t]{2}{*}{ Li3 et al. [42] } & L & $69(3-120)$ & $5 y, 52.0 \%$ & 0.805 \\
\hline & O & & $5 y, 53.4 \%$ & \\
\hline \multirow[t]{2}{*}{ Xu et al. [49] } & $\mathrm{L}$ & $58(0-129)$ & $5 y, 51.2 \%$ & 0.081 \\
\hline & $\mathrm{O}$ & $49.5(0-104.5)$ & $5 y, 46.7 \%$ & \\
\hline
\end{tabular}


Table 9 Systematic review of OS outcomes (Continued)

\begin{tabular}{llll}
\hline References & Group & Follow-up (mo.) & OS \\
\hline Inokuchi et al. [41] & $\mathrm{L}$ & $62.2(2.8-149.4)$ & $5 \mathrm{y}, 70 \%$ \\
Park et al. [43] & $\mathrm{O}$ & $62.2(4.4-130.4)$ & $5 \mathrm{y}, 73 \%$ \\
& $\mathrm{~L}$ & 38.2 & - \\
Chan et al. [45] & $\mathrm{O}$ & & \\
& $\mathrm{L}$ & 25 & 60 mo., $47 \%$ \\
Kinoshita et al. [46] & $\mathrm{O}$ & 35 & $60 \mathrm{mo} ., 39 \%$ \\
& $\mathrm{~L}$ & $3.4 \mathrm{y}(1.3-5.0)$ & $5 \mathrm{y}, 54.2 \%$ \\
& $\mathrm{O}$ & $3.5 \mathrm{y}(1.7-5.0)$ & $5 \mathrm{y}, 53.0 \%$ \\
\hline
\end{tabular}

OS over survival, DFS disease-free survival, NS not significant, - not report, $y$ year, mo. month

time, intraoperative blood loss, harvested lymph nodes, proximal and distal resection margin distance, time to first flatus, time to first oral intake, post-operative hospital stay, complication and mortality, rate of disease recurrence, and 5-year over survival.

The LG consumed significantly more time than OG, although we could get a wider operation field by applying LG. However, the operative process is more complicated and less flexible than OG; some reasons include the narrow operating field, restriction in the number of trocar [54], lacking of tactile sensation [55], insufficient training [56], the time for setting up the equipment, and the complexity of performing the esophagojejunostomy
[57], while LG combined with advanced techniques for systemic lymphadenectomy may be the main reason, which needs experienced surgeons. In terms of AGC, compared with gastrectomy alone, gastrectomy combined with systemic lymphadenectomy is more complicated. Meanwhile, compared with other laparoscopic surgery including laparoscopic colectomy and cholecystectomy, LG with lymphadenectomy is also more difficult because it is necessary to identified many important vessels and clear lymph node. Recently, some studies have indicated that the operative time could significantly reduce and reach a plateau after about 40 cases, and the operative time of LG is no longer than OG for extensive

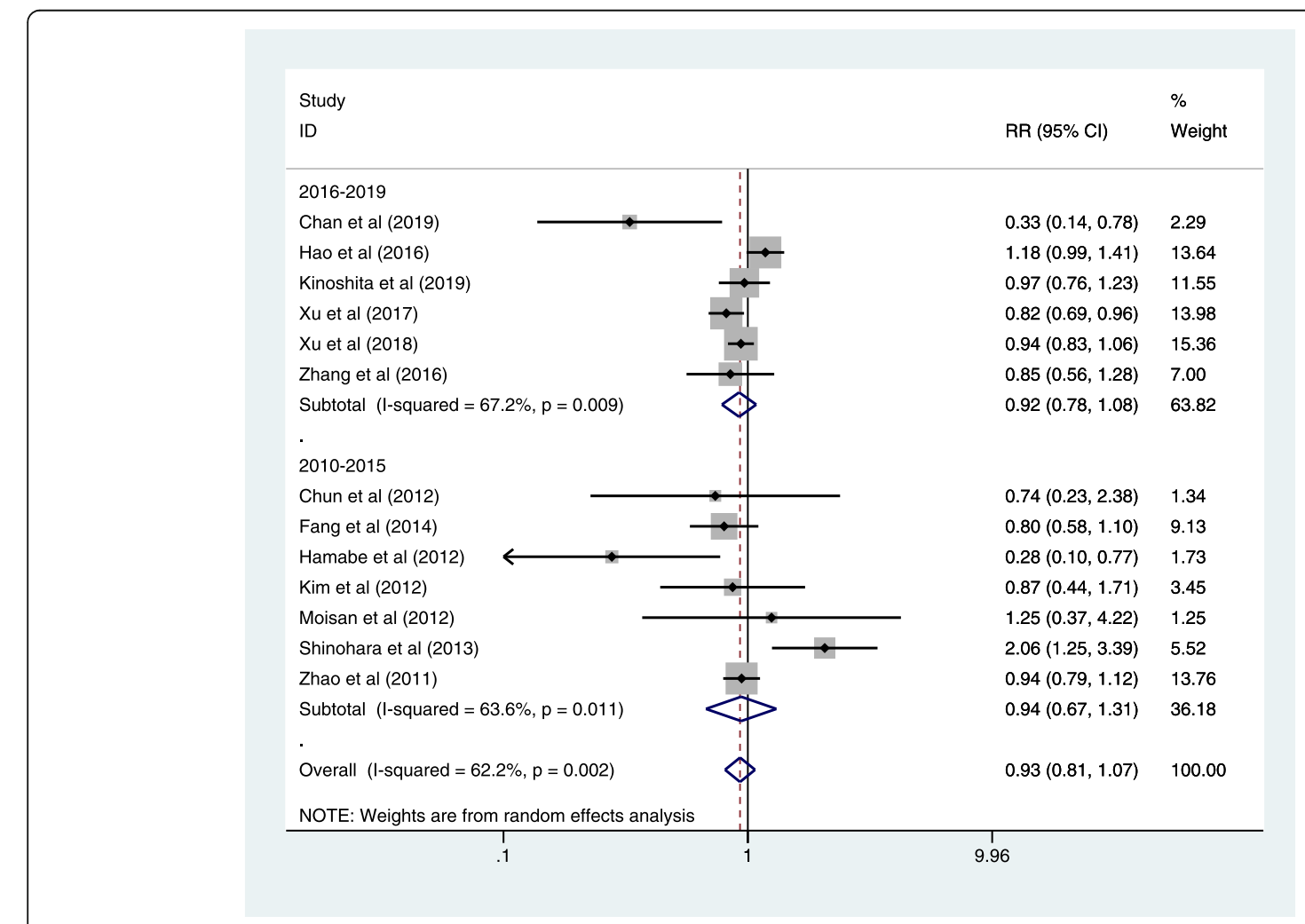

Fig. 6 Forest plot tumor recurrence 


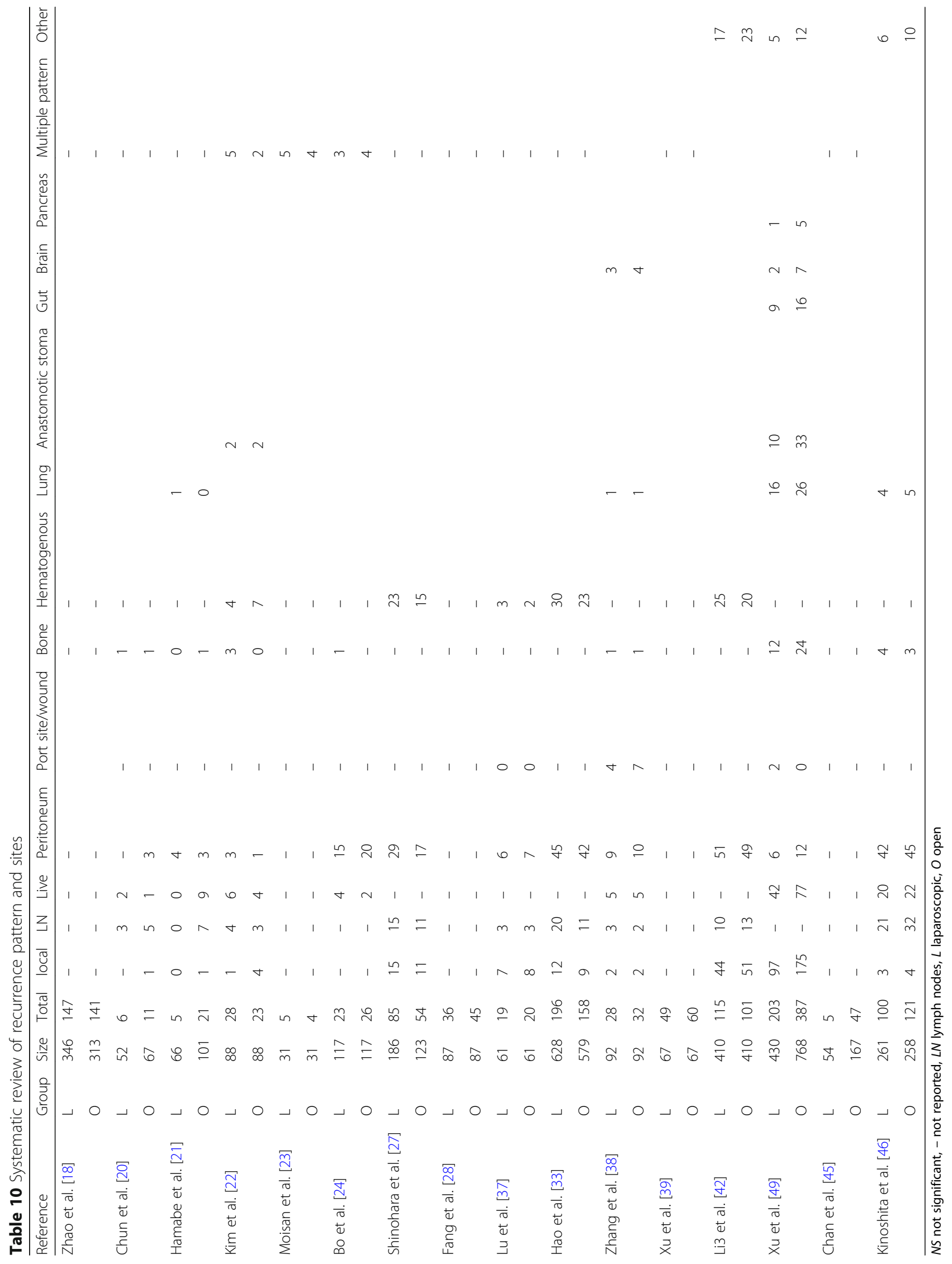


technical expertise [51, 58, 59]; meantime, some highquality studies reported postoperative morbidity has no significant difference between LG and OG, with LG leading to faster postoperative recovery [60]. With the development and improvement of laparoscopic techniques, the operative time will reduce and become shorter.

In spite of the operative time is longer, blood loss is significantly less in LG. For LG, by using the laparoscopic device such as ligatures and ultrasonic scalpel, we could get enlarged surgical vision to detect large and small vessels and expose vessel adequately, which contribute to small blood loss. The small amount of blood loss may contribute to a decreased blood transfusions, which could reduce the postoperative complication such as lung injury, volume overload, and pneumonia. Thus, small amount of blood loss has an impact on postoperative recovery and recurrence [61].

The postoperative complication is usually used to evaluate the surgical safety. The meta-analysis demonstrated that the overall postoperative complication rate of LG was significantly lower than OG; meantime, the wound problem and postoperative ileus were significantly less common than OG, which is in consistent with some previous meta-analysis $[62,63]$. For LG, the smaller surgical surface wound and less manual handling may account for less wound problem, and LG could reduce the intervention to microenvironment of abdominal cavity and intestinal serous membrane, which may decrease the rate of postoperative ileus. The rate of postoperative pneumonia was lower than OG with no significant difference. In terms of OG, some disadvantages may make it difficult to cough, which lead to respiratory complications such as pneumonia, including tension sutures, serious pain, and abdominal bandages, while patients in LG were related with less blood loss, less blood transfusion during surgery [64, 65], and less wound pain after surgery [66]. For other postoperative complications including pancreatitis/pancreatic leakage, intraabdominal bleeding, anastomotic bleeding, anastomotic stenosis, anastomotic leakage, duodenal stump leakage, abdominal infection, and lymphatic fistula, there were no significant differences.

With regard to the time to first flatus, the time to first oral intake, and post-operative hospital stay, the results were favoring for LG. LG is thought to be a less invasive procedure with smaller surgical incision and minimal gastrointestinal interference, so that the postoperative pain is less during recovery with a reduced inflammatory response and better glucose tolerance [67, 68], which has a direct impact on a quick recovery of bowel function, and a quick recovery represents earlier oral intake, earlier discharge, and shorter hospital stay. In other words, it is quicker to return to normal condition in LADG than ODG. The cost of laparoscopic surgery is higher for LG compared with OG, because of the costs of the disposable instruments, while Miura et al. [69] indicated that LADG was less expensive than ODG because hospital stay is shorter and additional costs can be offset by the lower charges for ward, meals, and nursing care.

In terms of oncological safety, in most previous studies, the number of HLNs is widely considered as the index of "quality" [70-75]; adequate LN dissection could reduce the possibility of recurrence and metastasis. The efficiency for lymphadenectomy is still the main concern; the efficiency represents surgical removal of fifteen lymph nodes is the minimum standard. In most previous studies and our present studies, the mean number of harvested lymph nodes was more than fifteen for LG. However, whether laparoscopy could reach the same result as open surgery is still controversial; a previous study showed that experienced surgeons could realize the radicality in lymphadenectomy if the operative time is not limited [76]. In the present meta-analysis, we discovered that the harvested lymph nodes have no significant difference, which indicated LG could retrieve as many LNs as did OG through the improvement in laparoscopy facilities and sufficient training. $\mathrm{D} 1+\alpha$ or $\beta$ dissection is now adequate for selected patients with early gastric cancer. With regard to advanced gastric cancer, whether D2 dissection is superior to D1 dissection remains controversial [77, 78]. D2 dissection could realize more radical lymphadenectomy than D1 dissection, whereas D2 dissection could increase the postoperative mobility and mortality because of the invasiveness. In East Asia, maybe the incidence is high so that Asian surgeons are familiar with gastric cancer and have a better understanding of surgical technique; D2 lymphadenectomy is generally accepted as the standard to treat AGC. The Japanese Gastric Cancer Association has presented D2 lymphadenectomy as the standard treatment of local AGC [79]. However, some western studies have reported no significant long-term advantage with higher operative morbidity and mortality rates after D2 lymphadenectomy [80-82], and western surgeons would like to perform D1 lymphadenectomy; many recent report have indicated that patients who underwent laparoscopic gastrectomy combined with systemic lymphadenectomy could get a good survival result by extensively trained western surgeons [83, 84]. Therefore, insufficient training of the laparoscopic gastrectomy combined with systemic lymphadenectomy may 
be the main reason for western surgeons. D2 dissection is an appropriate treatment for patients with advanced disease. Proximal esophageal and distal duodenal margins is also used to assess oncological adequacy, and proximal and distal margin distance could reflect the radicality of surgery, which is related with recurrence and OS and DFS in GC and other cancer [85]. Meantime, surgical margin is considered as an independent prognostic factor for GC. Our analyses also showed that there was no significant difference between the two groups; it indicated that LG is oncologically acceptable for proximal or distal located tumors.

Long-term outcome is the most useful endpoint to evaluate the oncological safety and effectiveness of surgery. Tumor recurrence and 5-year OS are usually used to evaluated the long-term outcome. Many studies have showed tumor recurrence was similar between the LADG and ODG [86, 87]; previous clinical studies and meta-analysis have revealed that there was no significant difference in the 5-year OS between LADG and ODG [89]. In our meta-analysis, we make subgroup analysis based on published year; there demonstrated no statistically significant difference between the two groups; however, the results of 5-year OS favor LG with significant difference between 2016 and 2020, and the tumor recurrence showed no significant difference between the two groups. In other words, at least, LG is not inferior to ODG in terms of oncologic outcomes, which is acceptable for treatment of AGC.

Some limitations exist that should not be neglected for this meta-analysis. Many studies related with the theme are non-randomized retrospective trials; therefore, we have analyzed both the RCTs and NRCTs to avoid lack of samples. Several drawbacks of methodology may lead to heterogeneity, although the study has no observed obvious heterogeneity. In many included studies, the patients with multiple tumor stages were incorporated into one group and included in a single survival curve; it will have a significant effect on the quality and results of the meta-analysis. More ongoing RCTs should be performed to resolve the problem in the future.

\section{Conclusion}

In conclusion, we make a systematic review of thirty-six studies to release LG could be considered to be expanded in treating AGC. Gastric cancer is difficult to diagnose early with a poor prognosis, and patients often do not realize until cancer progresses to middle and advanced stages. Our study has presented the safety and curability of LG, which indicated an encouraging result for LG to be widely accepted in the future. More ongoing RCTs comparing the LAG with OG should be recommended.

\section{Supplementary information}

Supplementary information accompanies this paper at https://doi.org/10. 1186/s12957-020-01888-7.

Additional file 1: Figure S1. Subgroup analysis based on clinical study type for lymph node dissection.

Additional file 2: Figure S2. Subgroup analysis based on the type of gastrectomy for lymph node dissection.

Additional file 3: Figure S3. Subgroup based on clinical study type for postoperative complications.

Additional file 4: Figure S4. Subgroup analysis based on the type of gastrectomy for postoperative complications.

Additional file 5: Figure S5. Subgroup analysis based on clinical study type for post-operative mortality.

Additional file 6: Figure S6. Subgroup analysis based on operative procedure for post-operative mortality.

Additional file 7: Figure S7. Subgroup analysis based on operative procedure for 5-year overall survival.

Additional file 8: Figure S8. Subgroup analysis based on operative procedure for tumor recurrence.

\section{Abbreviations}

LG: Laparoscopic gastrectomy; OG: Open gastrectomy; AGC: Advanced gastric cancer; 5-y OS: 5-Year over survival; RCT: Randomized controlled trials; NRCT: Retrospective studies; NOS: Newcastle-Ottawa Quality Assessment Scale; JCS: Jadad scale; RR: Relative risks; WMD: Weighted mean differences; HR: Hazard ratios; DG: Distal gastrectomy; TG: Total gastrectomy

\section{Acknowledgements}

We are grateful to the editor and reviewers for their constructive comments which led to improvements in this manuscript. We wish to thank everyone who helped with this study.

\section{Authors' contributions}

ZPZ and JHX designed and performed the study. ZPZ and LLL compiled the literature selection criteria and developed the literature search. JHX and WPY extracted the original data. JJZ and BRC performed the statistical analysis. WPY and JJZ processed the related figures and tables. ZPZ, JHX, and ZJH drafted the manuscript. All authors approved the final manuscript.

\section{Funding}

This study was supported by Project of Xiamen Scientific and Technological Plan (no. 3502Z20194005, 3502Z20184020)

Availability of data and materials

The datasets used and/or analyzed during the current study are available from the corresponding author upon reasonable request.

Ethics approval and consent to participate

Not applicable

\section{Consent for publication}

Not applicable

\section{Competing interests}

The authors declare that they have no competing interests.

Received: 11 April 2020 Accepted: 19 May 2020

Published online: 13 June 2020

\section{References}

1. Torre LA, Bray F, Siegel RL, Ferlay J, Lortet-Tieulent J, Jemal A. Global cancer statistics, 2012. CA Cancer J Clin. 2015;65:87-108. https://doi.org/10.3322/ caac. 21262.

2. Smith JK, McPhee JT, Hill JS, Whalen GF, Sullivan ME, Litwin DE, Anderson FA, Tseng JF. National outcomes after gastric resection for neoplasm. Arch Surg. 2007;142:387-93. https://doi.org/10.1001/archsurg.142.4.387. 
3. Kitano S, Iso Y, Moriyama M, Sugimachi K. Laparoscopy-assisted Billroth I gastrectomy. Surg Laparosc Endosc. 1994;4:146-8.

4. Kim HH, Hyung WJ, Cho GS, Kim MC, Han SU, Kim W, Ryu SW, Lee HJ, Song KY. Morbidity and mortality of laparoscopic gastrectomy versus open gastrectomy for gastric cancer: an interim report--a phase III multicenter, prospective, randomized trial (KLASS Trial). Ann Surg. 2010;251:417-20. https://doi.org/10.1097/SLA.0b013e3181cc8f6b.

5. Ryu KW, Kim YW, Lee JH, Nam BH, Kook MC, Choi IJ, Bae JM. Surgical complications and the risk factors of laparoscopy-assisted distal gastrectomy in early gastric cancer. Ann Surg Oncol. 2008;15:1625-31. https://doi.org/10. 1245/s10434-008-9845-x.

6. Hayashi H, Ochiai T, Shimada H, Gunji Y. Prospective randomized study of open versus laparoscopy-assisted distal gastrectomy with extraperigastric lymph node dissection for early gastric cancer. Surg Endosc. 2005;19:11726. https://doi.org/10.1007/s00464-004-8207-4.

7. Uyama I, Sugioka A, Matsui H, Fujita J, Komori Y, Hasumi A. Laparoscopic D2 lymph node dissection for advanced gastric cancer located in the middle or lower third portion of the stomach. Gastric Cancer. 2000;3:50-5.

8. Kim HH, Han SU, Kim MC, Hyung WJ, Kim W, Lee HJ, Ryu SW, Cho GS, Kim $\mathrm{CY}$, Yang HK, et al. Prospective randomized controlled trial (phase III) to comparing laparoscopic distal gastrectomy with open distal gastrectomy for gastric adenocarcinoma (KLASS 01). J Korean Surg Soc. 2013;84:123-30. https://doi.org/10.4174/jkss.2013.84.2.123.

9. Nakamura K, Katai H, Mizusawa J, Yoshikawa T, Ando M, Terashima M, Ito S, Takagi M, Takagane A, Ninomiya M, et al. A phase III study of laparoscopyassisted versus open distal gastrectomy with nodal dissection for clinical stage IA/IB gastric cancer (JCOG0912). Jpn J Clin Oncol. 2013;43:324-7. https://doi.org/10.1093/jjco/hys220.

10. Zou ZH, Zhao LY, Mou TY, Hu YF, Yu J, Liu H, Chen H, Wu JM, An SL, Li GX. Laparoscopic vs open D2 gastrectomy for locally advanced gastric cancer: a meta-analysis. World J Gastroenterol. 2014;20:16750-64. https://doi.org/10. 3748/wjg.v20.i44.16750

11. Wang W, Zhang X, Shen C, Zhi X, Wang B, Xu Z. Laparoscopic versus open total gastrectomy for gastric cancer: an updated meta-analysis. PLoS One. 2014;9:e88753. https://doi.org/10.1371/journal.pone.0088753.

12. Stang A. Critical evaluation of the Newcastle-Ottawa scale for the assessment of the quality of nonrandomized studies in meta-analyses. Eur J Epidemiol. 2010;25:603-5. https://doi.org/10.1007/s10654-010-9491-z.

13. Jadad AR, Moore RA, Carroll D, Jenkinson C, Reynolds DJ, Gavaghan DJ, McQuay HJ. Assessing the quality of reports of randomized clinical trials: is blinding necessary? Control Clin Trials. 1996;17:1-12. https://doi.org/10.1016/ 0197-2456(95)00134-4.

14. Huang JL, Wei HB, Zheng ZH, Wei B, Chen TF, Huang Y, Guo WP, Hu B. Laparoscopy-assisted D2 radical distal gastrectomy for advanced gastric cancer. Dig Surg. 2010;27:291-6. https://doi.org/10.1159/000281818.

15. Cai J, Wei D, Gao CF, Zhang CS, Zhang H, Zhao T. A prospective randomized study comparing open versus laparoscopy-assisted D2 radical gastrectomy in advanced gastric cancer. Dig Surg. 2011;28:331-7. https:// doi.org/10.1159/000330782

16. Scatizzi M, Kroning KC, Lenzi E, Moraldi L, Cantafio S, Feroci F. Laparoscopic versus open distal gastrectomy for locally advanced gastric cancer: a case-control study. Updat Surg. 2011;63:17-23. https://doi.org/10.1007/s13304-011-0043-1.

17. Shuang J, Qi S, Zheng J, Zhao Q, Li J, Kang Z, Hua J, Du J. A case-control study of laparoscopy-assisted and open distal gastrectomy for advanced gastric cancer. J Gastrointest Surg. 2011;15:57-62. https://doi.org/10.1007/ s11605-010-1361-1.

18. Zhao Y, Yu P, Hao Y, Qian F, Tang B, Shi Y, Luo H, Zhang Y. Comparison of outcomes for laparoscopically assisted and open radical distal gastrectomy with lymphadenectomy for advanced gastric cancer. Surg Endosc. 2011;25 2960-6. https://doi.org/10.1007/s00464-011-1652-y.

19. Chen QY, Huang CM, Lin JX, Zheng CH, Li P, Xie JW, Wang JB, Lu J. Laparoscopy-assisted versus open D2 radical gastrectomy for advanced gastric cancer without serosal invasion: a case control study. World J Surg Oncol. 2012;10:248. https://doi.org/10.1186/1477-7819-10-248.

20. Chun HT, Kim KH, Kim MC, Jung GJ. Comparative study of laparoscopyassisted versus open subtotal gastrectomy for pT2 gastric cancer. Yonsei Med J. 2012;53:952-9. https://doi.org/10.3349/ymj.2012.53.5.952.

21. Hamabe A, Omori T, Tanaka K, Nishida T. Comparison of long-term results between laparoscopy-assisted gastrectomy and open gastrectomy with D2 lymph node dissection for advanced gastric cancer. Surg Endosc. 2012;26: 1702-9. https://doi.org/10.1007/s00464-011-2096-0.
22. Kim KH, Kim MC, Jung GJ, Choi HJ, Jang JS, Kwon HC. Comparative analysis of five-year survival results of laparoscopy-assisted gastrectomy versus open gastrectomy for advanced gastric cancer: a case-control study using a propensity score method. Dig Surg. 2012;29:165-71. https://doi.org/10.1159/ 000338088 .

23. Moisan F, Norero E, Slako M, Varas J, Palominos G, Crovari F, Ibanez L, Perez G, Pimentel F, Guzman S, et al. Completely laparoscopic versus open gastrectomy for early and advanced gastric cancer: a matched cohort study. Surg Endosc. 2012;26:661-72. https://doi.org/10.1007/ s00464-011-1933-5.

24. Bo T, Peiwu Y, Feng Q, Yongliang Z, Yan S, Yingxue $H$, Huaxing L. Laparoscopy-assisted vs. open total gastrectomy for advanced gastric cancer: long-term outcomes and technical aspects of a case-control study. J Gastrointest Surg. 2013;17:1202-8. https://doi.org/10.1007/ s11605-013-2218-1.

25. Gordon AC, Kojima K, Inokuchi M, Kato K, Sugihara K. Long-term comparison of laparoscopy-assisted distal gastrectomy and open distal gastrectomy in advanced gastric cancer. Surg Endosc. 2013;27:462-70. https://doi.org/10.1007/s00464-012-2459-1.

26. Lin JX, Huang CM, Zheng CH, Li P, Xie JW, Wang JB, Lu J. Laparoscopyassisted gastrectomy with D2 lymph node dissection for advanced gastric cancer without serosa invasion: a matched cohort study from South China. World J Surg Oncol. 2013;11:4. https://doi.org/10.1186/1477-7819-11-4.

27. Shinohara T, Satoh S, Kanaya S, Ishida Y, Taniguchi K, Isogaki J, Inaba K, Yanaga K, Uyama I. Laparoscopic versus open D2 gastrectomy for advanced gastric cancer: a retrospective cohort study. Surg Endosc. 2013;27:286-94. https://doi.org/10.1007/s00464-012-2442-X.

28. Fang C, Hua J, Li J, Zhen J, Wang F, Zhao Q, Shuang J, Du J. Comparison of long-term results between laparoscopy-assisted gastrectomy and open gastrectomy with D2 lymphadenectomy for advanced gastric cancer. Am J Surg. 2014;208:391-6. https://doi.org/10.1016/j.amjsurg.2013.09.028.

29. Qiu JF, Yang B, Fang L, Li YP, Shi YJ, Yu XC, Zhang MC. Safety and efficacy of laparoscopy-assisted gastrectomy for advanced gastric cancer in the elderly. Int J Clin Exp Med. 2014;7:3562-7.

30. Cui M, Li Z, Xing J, Yao Z, Liu M, Chen L, Zhang C, Yang H, Zhang N, Tan F, et al. A prospective randomized clinical trial comparing D2 dissection in laparoscopic and open gastrectomy for gastric cancer. Med Oncol. 2015;32: 241. https://doi.org/10.1007/s12032-015-0680-1.

31. Wu LM, Jiang XJ, Lin QF, Jian CX. Comparative study of clinical efficacy of laparoscopy-assisted radical gastrectomy versus open radical gastrectomy for advanced gastric cancer. Genet Mol Res. 2015;14:3459-65. https://doi. org/10.4238/2015.April.15.9.

32. Zhang $Y$, Qi F, Jiang $Y$, Zhai H, Ji Y. Long-term follow-up after laparoscopic versus open distal gastrectomy for advanced gastric cancer. Int J Clin Exp Med. 2015:8:13564-70.

33. Hao Y, Yu P, Qian F, Zhao Y, Shi Y, Tang B, Zeng D, Zhang C. Comparison of laparoscopy-assisted and open radical gastrectomy for advanced gastric cancer: a retrospective study in a single minimally invasive surgery center. Medicine (Baltimore). 2016;95:e3936. https://doi.org/10.1097/MD. 0000000000003936.

34. Hu Y, Huang $C$, Sun $Y$, Su X, Cao H, Hu J, Xue Y, Suo J, Tao K, He X, et al. Morbidity and mortality of laparoscopic versus open D2 distal gastrectomy for advanced gastric cancer: a randomized controlled trial. J Clin Oncol. 2016:34:1350-7. https://doi.org/10.1200/JCO.2015.63.7215.

35. Li Q, Wang J, Zhang G, Wang J, Yang B, Zhang Z. Feasibility and safety comparison of laparoscopy-assisted versus open gastrectomy for advanced gastric carcinoma with D2 lymphadenectomy. Jpn J Clin Oncol. 2016;46: 323-8. https://doi.org/10.1093/jjco/hyw001.

36. Li Z, Shan F, Wang Y, Li S, Jia Y, Zhang L, Yin D, Ji J. Laparoscopic versus open distal gastrectomy for locally advanced gastric cancer after neoadjuvant chemotherapy: safety and short-term oncologic results. Surg Endosc. 2016;30:4265-71. https://doi.org/10.1007/s00464-015-4739-z.

37. Lu Y, Jiang B, Liu T. Laparoscopic versus open total gastrectomy for advanced proximal gastric carcinoma: a matched pair analysis. J BUON. 2016;21:903-8.

38. Zhang X, Sun F, Li S, Gao W, Wang Y, Hu SY. A propensity score-matched case-control comparative study of laparoscopic and open gastrectomy for locally advanced gastric carcinoma. J BUON. 2016;21:118-24.

39. Xu Y, Hua J, Li J, Shi L, Yuan J, Du J. Laparoscopic versus open gastrectomy for gastric cancer with serous invasion: long-term outcomes. J Surg Res. 2017;215:190-5. https://doi.org/10.1016/j.jss.2017.03.048. 
40. Zhang F, Lan Y, Tang B, Hao Y, Shi Y, Yu P. Comparative study of laparoscopy-assisted and open radical gastrectomy for stage T4a gastric cancer. Int J Surg. 2017;41:23-7. https://doi.org/10.1016/j.jisu.2017.01.116.

41. Inokuchi M, Nakagawa M, Tanioka T, Okuno K, Gokita K, Kojima K. Long- and short-term outcomes of laparoscopic gastrectomy versus open gastrectomy in patients with clinically and pathological locally advanced gastric cancer: a propensity-score matching analysis. Surg Endosc. 2018;32:735-42. https:// doi.org/10.1007/s00464-017-5730-7.

42. Li Z, Li B, Bai B, Yu P, Lian B, Zhao Q. Long-term outcomes of laparoscopic versus open D2 gastrectomy for advanced gastric cancer. Surg Oncol. 2018; 27:441-8. https://doi.org/10.1016/j.suronc.2018.05.022.

43. Park YK, Yoon HM, Kim YW, Park JY, Ryu KW, Lee YJ, Jeong O, Yoon KY, Lee $\mathrm{JH}$, Lee SE, et al. Laparoscopy-assisted versus open D2 distal gastrectomy for advanced gastric cancer: results from a randomized phase II multicenter clinical trial (COACT 1001). Ann Surg. 2018;267:638-45. https://doi.org/10. 1097/SLA.0000000000002168.

44. Shi Y, Xu X, Zhao Y, Qian F, Tang B, Hao Y, Luo H, Chen J, Yu P. Short-term surgical outcomes of a randomized controlled trial comparing laparoscopic versus open gastrectomy with D2 lymph node dissection for advanced gastric cancer. Surg Endosc. 2018;32:2427-33. https://doi.org/10.1007/ s00464-017-5942-X

45. Chan BYO, Yau KKW, Chan CKO. Totally laparoscopic versus open gastrectomy for advanced gastric cancer: a matched retrospective cohort study. Hong Kong Med J. 2019;25:30-7. https://doi.org/10.12809/ hkmj177150.

46. Kinoshita T, Uyama I, Terashima M, Noshiro H, Nagai E, Obama K, Tamamori Y, Nabae T, Honda M, Abe T, et al. Long-term outcomes of laparoscopic versus open surgery for clinical stage II/III gastric cancer: a multicenter cohort study in Japan (LOC-A Study). Ann Surg. 2019;269:887-94. https:// doi.org/10.1097/SLA.0000000000002768.

47. Lee HJ, Hyung WJ, Yang HK, Han SU, Park YK, An JY, Kim W, Kim HI, Kim HH, Ryu SW, et al. Short-term outcomes of a multicenter randomized controlled trial comparing laparoscopic distal gastrectomy with D2 lymphadenectomy to open distal gastrectomy for locally advanced gastric cancer (KLASS-02-RCT). Ann Surg. 2019. https://doi.org/ 10.1097/SLA.0000000000003217

48. Wang Z, Xing J, Cai J, Zhang Z, Li F, Zhang N, Wu J, Cui M, Liu Y, Chen L, et al. Short-term surgical outcomes of laparoscopy-assisted versus open D2 distal gastrectomy for locally advanced gastric cancer in North China: a multicenter randomized controlled trial. Surg Endosc. 2019;33:33-45. https:// doi.org/10.1007/s00464-018-6391-x.

49. Xu Y, Hua J, Li J, Shi L, Xue H, Shuang J, Du J. Long-term outcomes of laparoscopic versus open gastrectomy for advanced gastric cancer: a large cohort study. Am J Surg. 2019;217:750-6. https://doi.org/10.1016/j.amjsurg. 2018.07.012

50. Kim HG, Park JH, Jeong SH, Lee YJ, Ha WS, Choi SK, Hong SC, Jung EJ, Ju YT, Jeong CY, et al. Totally laparoscopic distal gastrectomy after learning curve completion: comparison with laparoscopy-assisted distal gastrectomy. J Gastric Cancer. 2013;13:26-33. https://doi.org/10.5230/jgc.2013.13.1.26.

51. Aurello P, Sagnotta A, Terrenato I, Berardi G, Nigri G, D'Angelo F, Ramacciato $\mathrm{G}$. Oncologic value of laparoscopy-assisted distal gastrectomy for advanced gastric cancer: a systematic review and meta-analysis. J Minim Access Surg. 2016;12:199-208. https://doi.org/10.4103/0972-9941.181283.

52. Ye LY, Liu DR, Li C, Li XW, Huang LN, Ye S, Zheng YX, Chen L. Systematic review of laparoscopy-assisted versus open gastrectomy for advanced gastric cancer. J Zhejiang Univ Sci B. 2013;14:468-78. https://doi.org/10. 1631/jzus.B1200197.

53. Amin AT, Gabr A, Abbas H. Laparoscopy assisted distal gastrectomy for $\mathrm{T} 1$ to T2 stage gastric cancer: a pilot study of three ports technique. Updat Surg. 2015;67:69-74. https://doi.org/10.1007/s13304-015-0279-2.

54. Wottawa CR, Cohen JR, Fan RE, Bisley JW, Culjat MO, Grundfest WS, Dutson EP. The role of tactile feedback in grip force during laparoscopic training tasks. Surg Endosc. 2013;27:1111-8. https://doi.org/10.1007/s00464-0122612-x.

55. Campo R, Puga M, Meier Furst R, Wattiez A, De Wilde RL. Excellence needs training "Certified programme in endoscopic surgery". Facts Views Vis Obgyn. 2014;6:240-4.

56. Fujiwara M, Kodera $Y$, Misawa K, Kinoshita M, Kinoshita T, Miura S, Ohashi N, Nakayama G, Koike M, Nakao A. Long term outcomes of early-stage gastric carcinoma patients treated with laparoscopy-assisted surgery. J Am Coll Surg. 2008;206:138-43. https://doi.org/10.1016/j.jamcollsurg.2007.07.013.
57. Hyung WJ, Song C, Cheong JH, Choi SH, Noh SH. Factors influencing operation time of laparoscopy-assisted distal subtotal gastrectomy: analysis of consecutive 100 initial cases. Eur J Surg Oncol. 2007;33:314-9. https://doi. org/10.1016/j.ejso.2006.11.010.

58. Hu WG, Ma JJ, Zang L, Xue P, Xu H, Wang ML, Lu AG, Li JW, Feng B, Zheng $\mathrm{MH}$. Learning curve and long-term outcomes of laparoscopy-assisted distal gastrectomy for gastric cancer. J Laparoendosc Adv Surg Tech A. 2014;24: 487-92. https://doi.org/10.1089/lap.2013.0570.

59. Yoo CH, Kim HO, Hwang SI, Son BH, Shin JH, Kim H. Short-term outcomes of laparoscopic-assisted distal gastrectomy for gastric cancer during a surgeon's learning curve period. Surg Endosc. 2009;23:2250-7. https://doi. org/10.1007/s00464-008-0315-0.

60. Vamvakas EC. Perioperative blood transfusion and cancer recurrence: metaanalysis for explanation. Transfusion. 1995;35:760-8.

61. Chen K, Xu XW, Mou YP, Pan Y, Zhou YC, Zhang RC, Wu D. Systematic review and meta-analysis of laparoscopic and open gastrectomy for advanced gastric cancer. World J Surg Oncol. 2013;11:182. https://doi.org/ 10.1186/1477-7819-11-182.

62. Xiong JJ, Nunes QM, Huang W, Tan CL, Ke NW, Xie SM, Ran X, Zhang H, Chen YH, Liu XB. Laparoscopic vs open total gastrectomy for gastric cancer: a meta-analysis. World J Gastroenterol. 2013;19:8114-32. https://doi.org/10. 3748/wjg.v19.i44.8114.

63. Ari Jutkowitz L. Blood transfusion in the perioperative period. Clin Tech Small Anim Pract. 2004;19:75-82. https://doi.org/10.1053/j.ctsap.2004.01.004.

64. Weber RS, Jabbour N, Martin RC 2nd. Anemia and transfusions in patients undergoing surgery for cancer. Ann Surg Oncol. 2008;15:34-45. https://doi. org/10.1245/s10434-007-9502-9.

65. Tanimura S, Higashino M, Fukunaga Y, Kishida S, Ogata A, Fujiwara Y, Osugi $H$. Respiratory function after laparoscopic distal gastrectomy--an index of minimally invasive surgery. World J Surg. 2006;30:1211-5. https://doi.org/10. 1007/s00268-005-0115-9.

66. Natsume T, Kawahira H, Hayashi H, Nabeya $Y$, Akai T, Horibe D, Shuto K, Akutsu Y, Matsushita K, Nomura F, et al. Low peritoneal and systemic inflammatory response after laparoscopy-assisted gastrectomy compared to open gastrectomy. Hepatogastroenterology. 2011;58:65962.

67. Kawamura H, Yokota R, Homma S, Kondo Y. Comparison of invasiveness between laparoscopy-assisted total gastrectomy and open total gastrectomy. World J Surg. 2009;33:2389-95. https://doi.org/10.1007/s00268009-0208-y.

68. Adachi Y, Shiraishi N, Ikebe K, Aramaki M, Bandoh T, Kitano S. Evaluation of the cost for laparoscopic-assisted Billroth I gastrectomy. Surg Endosc. 2001; 15:932-6. https://doi.org/10.1007/s004640090089.

69. Miura S, Kodera Y, Fujiwara M, Ito S, Mochizuki Y, Yamamura Y, Hibi K, Ito K, Akiyama S, Nakao A. Laparoscopy-assisted distal gastrectomy with systemic lymph node dissection: a critical reappraisal from the viewpoint of lymph node retrieval. J Am Coll Surg. 2004;198:933-8. https://doi.org/10.1016/j. jamcollsurg.2004.01.021.

70. Ikeda O, Sakaguchi Y, Toh Y, Oogaki K, Oki E, Minami K, Okamura T, Baba H. Evaluation of oncological adequacy of laparoscopic distal gastrectomy with special attention to lymph node dissection: a comparison with conventional open gastrectomy. Hepatogastroenterology. 2012;59:627-32. https://doi.org/ 10.5754/hge10089.

71. Du XH, Li R, Chen L, Shen D, Li SY, Guo Q. Laparoscopy-assisted D2 radical distal gastrectomy for advanced gastric cancer: initial experience. Chin Med J. 2009;122:1404-7.

72. Huscher C, Mingoli A, Sgarzini G, Sansonetti A, Piro F, Ponzano C, Brachini G Value of extended lymphadenectomy in laparoscopic subtotal gastrectomy for advanced gastric cancer. J Am Coll Surg. 2005;200:314. https://doi.org/ 10.1016/j.jamcollsurg.2004.10.024.

73. Cui M, Xing JD, Yang W, Ma YY, Yao ZD, Zhang N, Su XQ. D2 dissection in laparoscopic and open gastrectomy for gastric cancer. World J Gastroenterol. 2012;18:833-9. https://doi.org/10.3748/wjg.v18.i8.833.

74. Huscher CG, Mingoli A, Sgarzini G, Sansonetti A, Di Paola M, Recher A, Ponzano C. Laparoscopic versus open subtotal gastrectomy for distal gastric cancer: five-year results of a randomized prospective trial. Ann Surg. 2005; 241:232-7. https://doi.org/10.1097/01.sla.0000151892.35922.f2.

75. Lee SS, Kim $1 \mathrm{H}$. Are there any disbenefits to patients in choosing laparoscopic gastrectomy by an expert in open gastrectomy? Aspects of surgical outcome and radicality of lymphadenectomy. Chin Med J. 2013;126: 4247-53. 
76. Hartgrink $\mathrm{HH}$, van de Velde $\mathrm{CJ}$, Putter $\mathrm{H}$, Bonenkamp JJ, Klein Kranenbarg E, Songun I, Welvaart K, van Krieken JH, Meijer S, Plukker JT, et al. Extended lymph node dissection for gastric cancer: who may benefit? Final results of the randomized Dutch gastric cancer group trial. J Clin Oncol. 2004;22: 2069-77. https://doi.org/10.1200/JCO.2004.08.026.

77. McCulloch P, Niita ME, Kazi H, Gama-Rodrigues JJ. Gastrectomy with extended lymphadenectomy for primary treatment of gastric cancer. $\mathrm{Br} J$ Surg. 2005;92:5-13. https://doi.org/10.1002/bjs.4839.

78. Nakajima T. Gastric cancer treatment guidelines in Japan. Gastric Cancer. 2002;5:1-5. https://doi.org/10.1007/s101200200000.

79. Bonenkamp JJ, Hermans J, Sasako M, van de Velde CJ, Welvaart K, Songun I, Meyer S, Plukker JT, Van Elk P, Obertop H, et al. Extended lymph-node dissection for gastric cancer. N Engl J Med. 1999;340:908-14. https://doi.org/ 10.1056/NEJM199903253401202.

80. Hartgrink HH, van de Velde CJ, Putter H, Songun I, Tesselaar ME, Kranenbarg EK, de Vries JE, Wils JA, van der Bijl J, van Krieken JH, et al. Neo-adjuvant chemotherapy for operable gastric cancer: long term results of the Dutch randomised FAMTX trial. Eur J Surg Oncol. 2004;30:643-9. https://doi.org/10. 1016/j.jejso.2004.04.013.

81. Danielson H, Kokkola A, Kiviluoto T, Siren J, Louhimo J, Kivilaakso E, Puolakkainen P. Clinical outcome after D1 vs D2-3 gastrectomy for treatment of gastric cancer. Scand J Surg. 2007;96:35-40. https://doi.org/10. 1177/145749690709600107.

82. Sierra A, Regueira FM, Hernandez-Lizoain JL, Pardo F, Martinez-Gonzalez MA, J AC (2003) Role of the extended lymphadenectomy in gastric cancer surgery: experience in a single institution. Ann Surg Oncol 10: 219-226.

83. Songun I, Putter H, Kranenbarg EM, Sasako M, van de Velde CJ. Surgical treatment of gastric cancer: 15-year follow-up results of the randomised nationwide Dutch D1D2 trial. Lancet Oncol. 2010;11:439-49. https://doi.org/ 10.1016/S1470-2045(10)70070-X.

84. Eberlin LS, Tibshirani RJ, Zhang J, Longacre TA, Berry GJ, Bingham DB, Norton JA, Zare RN, Poultsides GA. Molecular assessment of surgicalresection margins of gastric cancer by mass-spectrometric imaging. Proc Natl Acad Sci U S A. 2014;111:2436-41. https://doi.org/10.1073/pnas. 1400274111

85. Ding J, Liao GQ, Liu HL, Liu S, Tang J. Meta-analysis of laparoscopy-assisted distal gastrectomy with D2 lymph node dissection for gastric cancer. J Surg Oncol. 2012;105:297-303. https://doi.org/10.1002/jso.22098.

86. Wang W, Li Z, Tang J, Wang M, Wang B, Xu Z. Laparoscopic versus open total gastrectomy with D2 dissection for gastric cancer: a meta-analysis. J Cancer Res Clin Oncol. 2013;139:1721-34. https://doi.org/10.1007/s00432013-1462-9.

87. Ohtani H, Tamamori Y, Noguchi K, Azuma T, Fujimoto S, Oba H, Aoki T, Minami M, Hirakawa K. Meta-analysis of laparoscopy-assisted and open distal gastrectomy for gastric cancer. J Surg Res. 2011;171:479-85. https:// doi.org/10.1016/j.jss.2010.04.008.

\section{Publisher's Note}

Springer Nature remains neutral with regard to jurisdictional claims in published maps and institutional affiliations.

Ready to submit your research? Choose BMC and benefit from:

- fast, convenient online submission

- thorough peer review by experienced researchers in your field

- rapid publication on acceptance

- support for research data, including large and complex data types

- gold Open Access which fosters wider collaboration and increased citations

- maximum visibility for your research: over $100 \mathrm{M}$ website views per year

At $\mathrm{BMC}$, research is always in progress.

Learn more biomedcentral.com/submissions 